\title{
NUMERICAL SCHEMES FOR CONSERVATION LAWS VIA HAMILTON-JACOBI EQUATIONS
}

\author{
L. CORRIAS, M. FALCONE, AND R. NATALINI
}

\begin{abstract}
We present some difference approximation schemes which converge to the entropy solution of a scalar conservation law having a convex flux. The numerical methods described here take their origin from approximation schemes for Hamilton-Jacobi-Bellman equations related to optimal control problems and exhibit several interesting features: the convergence result still holds for quite arbitrary time steps, the main assumption for convergence can be interpreted as a discrete analogue of Oleinik's entropy condition, numerical diffusion around the shocks is very limited. Some tests are included in order to compare the performances of these methods with other classical methods (Godunov, TVD).
\end{abstract}

\section{INTRODUCTION}

We are interested in the approximation of the entropy weak solutions in the sense of Kružkov [15] of the following scalar conservation law:

$$
\begin{cases}u_{t}+f(u)_{x}=0 & \text { in } \mathbb{R} \times(0, \infty), \\ u(x, 0)=u_{0}(x) & \text { in } \mathbb{R} .\end{cases}
$$

The connections between this problem and the theory of generalized solutions (see, e.g., $[7,6,8,16])$ to the first-order Hamilton-Jacobi equation

$$
\begin{cases}v_{t}+f\left(v_{x}\right)=0 & \text { in } \mathbb{R} \times(0, \infty), \\ v(x, 0)=v_{0}(x) & \text { in } \mathbb{R}\end{cases}
$$

are known. Roughly speaking, if $v$ is a viscosity solution of $(\mathrm{HJ})$, then $u:=v_{x}$ is an entropy solution of $(\mathrm{CL})$ (for the precise results see $\S 2$ ). This relation has been used also for numerical purposes in order to derive schemes for $(\mathrm{HJ})$ from the large collection of methods for $(\mathrm{CL})$. In fact, it has been shown that integrating a scheme converging to the entropy solution of (CL) (called CLscheme in the sequel), one can obtain a scheme converging to the viscosity solution of $(\mathrm{HJ})(\mathrm{HJ}$-scheme in the sequel). This technique has been applied by several authors; e.g., Kružkov in [17] and Crandall and Lions in [9] have studied numerical methods derived from first-order schemes for $(\mathrm{CL})$, Osher

Received by the editor March 30, 1992 and, in revised form, February 10, 1993 and June 18, 1993.

1991 Mathematics Subject Classification. Primary 65M06; Secondary 35L65.

Key words and phrases. Difference approximation, conservation laws, entropy solutions, Hamilton-Jacobi equations. 
and Sethian in [25] have obtained schemes for (HJ) starting from ENO schemes (see also the recent paper of Lions and Souganidis [22] on the convergence of MUSCL schemes). We also use this relation for numerical purposes but in the opposite direction. The numerical approximation of $(\mathrm{HJ})$ has the advantage that we deal with more regular solutions (typically they are locally Lipschitz continuous) and we take a discrete derivative in $x$ on the numerical solution of $(\mathrm{HJ})$ to go back to $u$. Another advantage is that the expressions of the schemes for $(\mathrm{HJ})$ are relatively simple in comparison with their analogs for (CL) (see §5). Our main result is the following. Assume we have a numerical scheme approximating the (unique) viscosity solution $v$ of $(\mathrm{HJ})$, where the Hamiltonian $f$ is a $\mathscr{C}^{1}$ convex real function on $\mathbb{R}$ and $v_{0} \in W^{1, \infty}(\mathbb{R})$ (we will comment on the nonconvex case in Remark 5.1). Let $\Delta x$ and $\Delta t$ be respectively the space and time steps and set, for some fixed $N \in \mathbb{N}, T:=N \Delta t$. Let $v_{j}^{n}$ be a numerical approximation of $v$ at $(j \Delta x, n \Delta t)$, for $j \in \mathbb{Z}$ and $n \in\{0, \ldots, N\}$, and let us define, for example,

$$
u_{j}^{n}:=\frac{v_{j+1}^{n}-v_{j}^{n}}{\Delta x} .
$$

We define on $\mathbb{R} \times(0, T)$ the piecewise constant function $u_{\Delta}$ as follows:

$u_{\Delta}(x, t):=u_{j}^{n} \quad$ if $(x, t) \in\left[\left(j-\frac{1}{2}\right) \Delta x,\left(j+\frac{1}{2}\right) \Delta x\right) \times[n \Delta t,(n+1) \Delta t)$,

and we do the same for $v_{\Delta}$.

Theorem 1.1. Assume that for any $t \in(0, T)$, the sequence $v_{\Delta}$ converges in $L^{\infty}(\mathbb{R})$ to the viscosity solution of $(H J)$ as $\Delta x$ and $\Delta t$ go to zero, and the following properties hold:

(i) there exists a constant $C_{1}$ such that for any $j \in \mathbb{Z}$ and for any integer $n \in\{0, \ldots, N\}$

$$
\left|\frac{v_{j+1}^{n}-v_{j}^{n}}{\Delta x}\right| \leq C_{1}
$$

(ii) there exists a constant $C_{2}$ such that for any $j \in \mathbb{Z}$ and for any integer $n \in\{0, \ldots, N\}$

$$
\frac{v_{j+1}^{n}-2 v_{j}^{n}+v_{j-1}^{n}}{\Delta x^{2}} \leq C_{2} .
$$

Then for any $t \in(0, T)$ the sequence $u_{\Delta}$ converges in $L_{\text {loc }}^{1}(\mathbb{R})$ to the entropy solution $u$ of $(C L)$.

Notice that the assumption (i) only implies the weak convergence of $u_{\Delta}$ to $u$. Since assumption (ii) is a discrete equivalent of the celebrated Oleinik's entropy condition, this result can be stated saying that from an "entropic" scheme for viscosity solutions satisfying a bound on the discrete $x$-derivatives one can obtain, by (1.1), a scheme which converges strongly to the entropy solution of (CL). Notice that the forward difference in (1.1) is taken just for simplicity but that the result is still valid when using centered or backward differences. For initial data with compact support for (CL) the condition (1.2) can be dropped since it is implied by (1.3) (see Proposition 4.2). 
An example of methods satisfying assumptions (i), (ii) is given by a class of schemes studied by Falcone and Giorgi [11] (see also [10] and references therein). These schemes have been developed for the Hamilton-Jacobi-Bellman equation related to a finite horizon optimal control problem but can be adapted to $(\mathrm{HJ})$. Since they can be written in the form

$$
v_{j}^{n}=\min _{\alpha \in \mathbb{R}}\left\{v^{n-1}(j \Delta x-\alpha \Delta t)+\Delta t f^{*}(\alpha)\right\},
$$

where $f^{*}$ is the Legendre transform of $f$, one can interpret them as a discrete version of the Lax-Oleinik-Hopf representation formulas (see [19, 23, 14], and [21]) for the viscosity solutions of $(\mathrm{HJ})$, see also [24]. An interesting feature of these schemes is that they do not work on a fixed number of nodes, since the stencil depends on the ratio $\Delta t / \Delta x$ which may be nonconstant. Choosing a large time step will only increase the width of the stencil in such a way that the domain of dependence of the conservation law will always be contained in the numerical domain of dependence. Naturally, this will require a larger number of operations at each time step but, as we will show in the sequel, the global CPU time necessary to obtain accurate results is even lower than that needed by some classical schemes (see $\S 6$ ). The $\mathrm{HJ}$-schemes produce accurate approximate solutions both for smooth and for discontinuous solutions. We refer to [12] and [20] for a study and numerical experiments related to other difference schemes with large time step.

The outline of the paper is as follows. In $\S 2$ we review the relations between (CL) and (HJ), establishing some results about the precise correspondence between entropy solutions and viscosity solutions. In $\S 3$ we consider general classes of approximation schemes for both problems and prove the relations between them. Section 4 is devoted to the proof of our main convergence result, Theorem 1.1. In $\S 5$ we study the schemes derived from optimal control problems and prove that they satisfy the assumptions of the general convergence theorem. Some remarks on the CFL condition, the local truncation error and other properties of $\mathrm{HJ}$-schemes are also included in this section. Finally, $\S 6$ is devoted to numerical results for some typical examples. We compare the accuracy of our methods with other more classical schemes (Godunov, TVD) and we compute the approximate rate of convergence obtained in our tests.

\section{PReliminaries: Relation BeTWEen CONSERVATION LAWS AND HAMILTON-JACOBI EQUATIONS}

In this section we shall present for completeness some simple results about the equivalence of $(\mathrm{HJ})$ and $(\mathrm{CL})$ from an analytical point of view. First we quote a preliminary result from [21, Theorem 16.1, p. 268].

Proposition 2.1. Let $f \in C(\mathbb{R})$ and assume $v \in W^{1, \infty}(\mathbb{R} \times(0, T))$ to be a solution of $(H J)$. Then $u:=v_{x}$ is a weak solution of $(C L)$.

Our first result is the following

Theorem 2.2. Let $f \in C^{1}(\mathbb{R}), v_{0} \in W^{1, \infty}(\mathbb{R})$. If $v \in W^{1, \infty}(\mathbb{R} \times(0, T))$ is the (unique) viscosity solution of $(H J)$, then $u:=v_{x}$ is the (unique) entropy solution of $(C L)$. 
Proof. As proved in [9], the viscosity solution $v$ is the limit as $\varepsilon \rightarrow 0^{+}$in $L^{\infty}(\mathbb{R} \times(0, T))$ of the regular solutions $v^{\varepsilon}$ of the following problems:

$$
\begin{cases}v_{t}^{\varepsilon}+f\left(v_{x}^{\varepsilon}\right)=\varepsilon v_{x x}^{\varepsilon} & \text { in } \mathbb{R} \times(0, T), \\ v^{\varepsilon}(x, 0)=v_{0}(x) & \text { in } \mathbb{R} .\end{cases}
$$

Hence, we have for any $\varphi \in C_{0}^{\infty}(\mathbb{R} \times(0, T))$

$$
\begin{aligned}
\lim _{\varepsilon \rightarrow 0} \int_{0}^{T} \int_{\mathbf{R}} v_{x}^{\varepsilon} \varphi \mathrm{d} x \mathrm{~d} t & =-\lim _{\varepsilon \rightarrow 0} \int_{0}^{T} \int_{\mathbb{R}} v^{\varepsilon} \varphi_{x} \mathrm{~d} x \mathrm{~d} t \\
& =-\int_{0}^{T} \int_{\mathbb{R}} v \varphi_{x} \mathrm{~d} x \mathrm{~d} t=\int_{0}^{T} \int_{\mathbb{R}} v_{x} \varphi \mathrm{d} x \mathrm{~d} t .
\end{aligned}
$$

Obviously, the function $u^{\varepsilon}:=v_{x}^{\varepsilon}$ solves the derived problem

$$
\begin{cases}u_{t}^{\varepsilon}+f\left(u^{\varepsilon}\right)_{x}=\varepsilon u_{x x}^{\varepsilon} & \text { in } \mathbb{R} \times(0, T), \\ u^{\varepsilon}(x, 0)=v_{0 x}(x) & \text { in } \mathbb{R},\end{cases}
$$

and, according to [15], the sequence $u^{\varepsilon}$ converges in $L_{\text {loc }}^{1}(\mathbb{R} \times(0, T))$, as $\varepsilon \rightarrow 0$, to the entropy solution $u$ of $(\mathrm{CL})$.

Then, for any $\varphi \in C_{0}^{\infty}(\mathbb{R} \times(0, T))$,

Consequently,

$$
\lim _{\varepsilon \rightarrow 0} \int_{0}^{T} \int_{\mathbf{R}} u^{\varepsilon} \varphi \mathrm{d} x \mathrm{~d} t=\int_{0}^{T} \int_{\mathbf{R}} u \varphi \mathrm{d} x \mathrm{~d} t
$$

and $v_{x}=u$ a.e. in $\mathbb{R} \times(0, T)$.

$$
\int_{0}^{T} \int_{\mathbb{R}} v_{x} \varphi \mathrm{d} x \mathrm{~d} t=\int_{0}^{T} \int_{\mathbb{R}} u \varphi \mathrm{d} x \mathrm{~d} t
$$

A converse of these results also holds true:

Proposition 2.3. Let $f \in C(\mathbb{R})$ and assume $u \in L_{\text {loc }}^{\infty}(\mathbb{R} \times(0, T))$ to be a weak solution of $(C L)$. Let

$$
v(x, t):=\int_{\alpha}^{x} u(\xi, t) \mathrm{d} \xi
$$

for a fixed $\alpha \in \mathbb{R}$. Then $v \in W_{\mathrm{loc}}^{1, \infty}(\mathbb{R} \times(0, T))$, and $v$ is a solution of $(H J)$ almost everywhere.

Proof. Since $u \in L_{\text {loc }}^{\infty}(\mathbb{R} \times(0, T))$, there exists a set $A \subseteq(0, T)$, with Lebesgue measure $m(A)=0$, such that for any $t \in(0, T) \backslash A, u$ is defined a.e. on $\mathbb{R}$ and $u(\cdot, t) \in L_{\text {loc }}^{\infty}(\mathbb{R})$. Then for such values of $t, v(\cdot, t) \in L_{\text {loc }}^{\infty}(\mathbb{R})$. Moreover, for any $t \in(0, T) \backslash A$ and any $\varphi \in C_{0}^{\infty}(\mathbb{R} \times(0, T))$,

$$
\begin{aligned}
\int_{\mathbf{R}} v(x, t) \varphi_{x}(x, t) \mathrm{d} x & =\int_{\mathbf{R}}\left[\int_{\alpha}^{x} u(\xi, t) \mathrm{d} \xi\right] \varphi_{x}(x, t) \mathrm{d} x \\
& =-\int_{\mathbf{R}} u(x, t) \varphi(x, t) \mathrm{d} x .
\end{aligned}
$$

Thus, integrating on $(0, T)$, one has $u=v_{x}$ in the sense of distribution and almost everywhere. Since $u$ is a weak solution of $(\mathrm{CL})$, we have, for any $\varphi \in$ $C_{0}^{\infty}(\mathbb{R} \times(0, T))$,

$$
\begin{aligned}
\int_{0}^{T} \int_{\mathbf{R}} f(u) \varphi_{x} \mathrm{~d} x \mathrm{~d} t & =-\int_{0}^{T} \int_{\mathbf{R}} u \varphi_{t} \mathrm{~d} x \mathrm{~d} t \\
& =-\int_{0}^{T} \int_{\mathbf{R}} v_{x} \varphi_{t} \mathrm{~d} x \mathrm{~d} t=+\int_{0}^{T} \int_{\mathbf{R}} v \varphi_{t x} \mathrm{~d} x \mathrm{~d} t .
\end{aligned}
$$


So there exists $v_{t}$ in the sense of distribution, and $v_{t}=-f(u)=-f\left(v_{x}\right)$. Therefore, $v \in W_{\text {loc }}^{1, \infty}(\mathbb{R} \times(0, T))$, and $v$ is a solution almost everywhere of (HJ).

For any $u \in C\left([0, T] ; L^{1}(\mathbb{R})\right)$, we set

$$
v(x, t):=\int_{-\infty}^{x} u(\xi, t) \mathrm{d} \xi .
$$

Therefore, for any $t \in(0, T)$, the function $v(\cdot, t)$, is absolutely continuous and $v_{x}=u$ a.e.

Theorem 2.4. Let $f \in C^{1}(\mathbb{R})$ and $u_{0} \in L^{\infty}(\mathbb{R}) \cap L^{1}(\mathbb{R})$. Assume that $u \in$ $L^{\infty}(\mathbb{R} \times(0, T)) \cap C\left([0, T] ; L^{1}(\mathbb{R})\right)$ is the (unique) entropy solution of $(\mathrm{CL})$; then the function $v$ given by (2.3) is the (unique) viscosity solution of $(H J)$, for $v_{0}(x):=\int_{-\infty}^{x} u_{0}(\xi) \mathrm{d} \xi$.

Proof. Since $u \in C\left([0, T] ; L^{1}((\mathbb{R}))\right.$, we have that $v \in L^{\infty}(\mathbb{R} \times[0, T])$. As in the previous proposition, it is easy to show that $v \in W^{1, \infty}(\mathbb{R} \times(0, T))$ and that it is a solution almost everywhere of $(\mathrm{HJ})$. Also,

$$
\lim _{t \rightarrow 0}\left|v(x, t)-v_{0}(x)\right| \leq \lim _{t \rightarrow 0} \int_{-\infty}^{x}\left|u(\xi, t)-u_{0}(\xi)\right| \mathrm{d} \xi=0 .
$$

Now, suppose $v$ is not the viscosity solution of $(\mathrm{HJ})$, and denote by $\bar{v}$ the (unique) actual viscosity solution. So, by Theorem $2.2, \bar{v}_{x}$ is the unique entropy solution of $(\mathrm{CL})$ and therefore, for any $\varphi \in C_{0}^{\infty}(\mathbb{R} \times(0, T))$,

$$
\iint(v-\bar{v}) \varphi_{x} \mathrm{~d} x \mathrm{~d} t=0 .
$$

Hence, the conclusion follows from the arbitrariness of $\varphi$.

Remark 2.1. It would be interesting to prove this analytical equivalence without using the known results about existence, uniqueness, and convergence of the viscosity approximation. As far as we know, this proof has been obtained by the viscosity approximation only in the convex case and in the case of piecewise regular solutions (see [8]). More recently, Caselles [4] has proved the equivalence in a direct way for the stationary problem. It is probably possible to extend his methods to deal with the time-dependent problem.

\section{Relations between CL-schemes and HJ-schemes}

Here we consider the numerical aspects of the correspondence between entropy solutions of a conservation law and viscosity solutions of a HamiltonJacobi equation.

To approximate the solution $u$ of $(\mathrm{CL})$, we consider the class of finite difference schemes depending on $(2 p+1)$ grid points and written in conservation form, i.e.,

$$
u_{j}^{n+1}=u_{j}^{n}-\frac{\Delta t}{\Delta x}\left[\bar{f}_{j+\frac{1}{2}}^{n}-\bar{f}_{j-\frac{1}{2}}^{n}\right] .
$$

Here, $\Delta x$ and $\Delta t$ are the mesh sizes, $u_{j}^{n}$ is the value of the approximation of the solution $u$ at the grid node $(j \Delta x, n \Delta t)$, for $j \in \mathbb{Z}, n \in\{0, \ldots, N\}$ 
and $\bar{f}_{j+\frac{1}{2}}^{n}=\bar{f}\left(u_{j-p+1}^{n}, \ldots, u_{j+p}^{n}\right)$ is the numerical flux, where $\bar{f}: \mathbb{R}^{2 p} \rightarrow \mathbb{R}$ is a regular function.

For $\Delta x$ and $\Delta t$ fixed, the approximate solution is given by

$$
u_{\Delta}(x, t):=u_{j}^{n} \text { for }(x, t) \in I_{j, n},
$$

where, for $j \in \mathbb{Z}, n \in\{0, \ldots, N\}$,

$$
I_{j, n}:=\left[\left(j-\frac{1}{2}\right) \Delta x,\left(j+\frac{1}{2}\right) \Delta x\right) \times[n \Delta t,(n+1) \Delta t) .
$$

We assume for simplicity

$$
\left\{u_{j}^{n}\right\}_{j \in \mathbb{Z}} \in l_{1}, \quad \forall n \geq 0 .
$$

From (3.1) it is enough to assume (3.2) only for $n=0$.

Now we set, for $0 \leq n \leq N$,

$$
v_{j}^{n}:=\sum_{l=-\infty}^{j-1} u_{l}^{n} \Delta x .
$$

So, we can reverse this relation to obtain

$$
u_{j}^{n}=\frac{v_{j+1}^{n}-v_{j}^{n}}{\Delta x},
$$

and therefore, by using again (3.1), we obtain a finite difference $(2 p+1)$-point conservative scheme which approximates $(\mathrm{HJ})$, namely

$$
v_{j}^{n+1}=v_{j}^{n}-\Delta t \bar{f}\left(\frac{v_{j-p+1}^{n}-v_{j-p}^{n}}{\Delta x}, \ldots, \frac{v_{j+p}^{n}-v_{j+p-1}^{n}}{\Delta x}\right) .
$$

The approximate solution for $(\mathrm{HJ})$ is then given by

$$
v_{\Delta}(x, t):=v_{j}^{n} \text { for }(x, t) \in I_{j, n} .
$$

Theorem 3.1. If for any $t \in(0, T)$, the sequence $u_{\Delta}(\cdot, t)$ as $\Delta x$ and $\Delta t$ go to zero, converges in $L^{1}(\mathbb{R})$ to the entropy solution $u$ of $(C L)$, then, for any $t \in(0, T)$, the sequence $v_{\Delta}(\cdot, t)$ converges in $L^{\infty}(\mathbb{R})$ to the viscosity solution $v$ of $(H J)$.

Proof. For any $(x, t) \in \mathbb{R} \times(0, T)$ let $j \in \mathbb{Z}, n \in\{0, \ldots, N\}$ be such that $(x, t) \in I_{j, n}$. Since from Theorem 2.4 the function $v$ given by $(2.3)$ is the viscosity solution of $(\mathrm{HJ})$, we have

$$
\begin{aligned}
\left|v_{\Delta}(x, t)-v(x, t)\right|=\left|v_{j}^{n}-\int_{-\infty}^{x} u(\xi, t) \mathrm{d} \xi\right| \\
=\left|\sum_{l=-\infty}^{j-1} u_{j}^{n} \Delta x-\int_{-\infty}^{x} u(\xi, t) \mathrm{d} \xi\right| \\
=\left|\int_{-\infty}^{(j-1 / 2) \Delta x}\left(u_{\Delta}(\xi, t)-u(\xi, t)\right) \mathrm{d} \xi-\int_{(j-1 / 2) \Delta x}^{x} u(\xi, t) \mathrm{d} \xi\right| .
\end{aligned}
$$

Hence,

$$
\left\|v_{\Delta}(\cdot, t)-v(\cdot, t)\right\|_{L^{\infty}(\mathbf{R})} \leq\left\|u_{\Delta}(\cdot, t)-u(\cdot, t)\right\|_{L^{1}(\mathbf{R})}+\Delta x\|u\|_{L^{\infty}(\mathbf{R} \times(0, T))},
$$

and the theorem is proved. 
Remark 3.1. The extension of Theorem 3.1 to the case when (3.2) does not hold is straightforward. Notice that the conservation form (3.1) allows the simple expression (3.5), but in principle Theorem 3.1 holds true for any finite difference scheme.

\section{A gENERAL CONVERGENCE RESULT}

In order to obtain numerical schemes for conservation laws from those for Hamilton-Jacobi equations, we need to reverse the arguments of the preceding section.

Let $v_{j}^{n}$ be the approximation of the solution $v$ of $(\mathrm{HJ})$ at the grid point $(j \Delta x, n \Delta t)$, given by a general finite difference scheme

$$
v_{j}^{n+1}=F\left(v_{j-p}^{n}, \ldots, v_{j+p}^{n}\right),
$$

and define $u_{j}^{n}$, for example, as in (1.1). As above, $u_{\Delta}$ and $v_{\Delta}$ will denote the corresponding approximate solutions.

We recall that, if the function $f$ is strictly convex and $v$ is the viscosity solution of $(\mathrm{HJ})$, there exists a constant $K$ such that, for any $t>0$,

$$
v_{x x} \leq K \quad \text { in } \mathscr{D}^{\prime}(\mathbb{R} \times(0, T))
$$

if the same inequality holds true at $t=0$ (see [21, Theorem 16.1, p. 268] for a proof).

Lemma 4.1. Assume $v \in W^{1, \infty}(\mathbb{R})$ and suppose there exists a constant $K$ such that

$$
v_{x x} \leq K \quad \text { in } \mathscr{D}^{\prime}(\mathbb{R})
$$

Then, for any $h$ and any $x \in \mathbb{R}$,

$$
\frac{v(x+h)-2 v(x)+v(x-h)}{h^{2}} \leq K .
$$

The proof is omitted.

Proof of Theorem 1.1. It is clearly sufficient to work with the more convenient convergence in $L_{\text {loc }}^{2}$. Let $v$ be the viscosity solution of $(\mathrm{HJ})$. We set

$$
\tilde{u}_{\Delta}(x, t):=\frac{v((j+1) \Delta x, n \Delta t)-v(j \Delta x, n \Delta t)}{\Delta x} \text { for }(x, t) \in I_{j, n} .
$$

Let $I$ be any fixed bounded interval of $\mathbb{R}$. We have

$$
\begin{aligned}
\int_{I}\left|u_{\Delta}(x, t)-u(x, t)\right|^{2} \mathrm{~d} x \leq & 2 \int_{I}\left|u_{\Delta}(x, t)-\tilde{u}_{\Delta}(x, t)\right|^{2} \mathrm{~d} x \\
& +2 \int_{I}\left|\tilde{u}_{\Delta}(x, t)-u(x, t)\right|^{2} \mathrm{~d} x \\
=: & 2\left(I_{1}+I_{2}\right) .
\end{aligned}
$$

First step: the estimate of $I_{1}$. Let $S=\left\{j_{1}, \ldots, j_{m}\right\} \subseteq \mathbb{Z}$ be such that

$$
I \cap\left[\left(j_{i}-\frac{1}{2}\right) \Delta x,\left(j_{i}+\frac{1}{2}\right) \Delta x\right) \neq \varnothing
$$


for any $j_{i} \in S$. Then, if $t \in[n \Delta t,(n+1) \Delta t)$, we have

$$
\begin{aligned}
I_{1} & =\int_{I}\left|u_{\Delta}(x, t)-\tilde{u}_{\Delta}(x, t)\right|^{2} \mathrm{~d} x \\
& \leq \sum_{j \in S}\left|\frac{v_{j+1}^{n}-v_{j}^{n}}{\Delta x}-\frac{v((j+1) \Delta x, n \Delta t)-v(j \Delta x, n \Delta t)}{\Delta x}\right|^{2} \Delta x .
\end{aligned}
$$

With the notation $w_{j}^{n}:=v_{j}^{n}-v(j \Delta x, n \Delta t)$, the above inequality becomes

$$
I_{1} \leq \sum_{j \in S}\left|\frac{\Delta_{x}^{+} w_{j}^{n}}{\Delta x}\right|^{2} \Delta x,
$$

where $\Delta_{x}^{+}$is the forward first difference operator. Thus, setting $S^{\prime}=S \backslash\left\{j_{1}\right\}$, we have

$$
\begin{aligned}
& I_{1} \leq \sum_{j \in S}\left(w_{j+1}^{n}-w_{j}^{n}\right) \frac{\Delta_{x}^{+} w_{j}^{n}}{\Delta x^{2}} \Delta x \\
&=w_{j_{m}+1}^{n} \frac{\Delta_{x}^{+} w_{j_{m}}^{n}}{\Delta x}-w_{j_{1}}^{n} \frac{\Delta_{x}^{+} w_{j_{1}}^{n}}{\Delta x}-\sum_{j \in S^{\prime}} w_{j}^{n} \frac{\Delta_{x}^{+} w_{j}^{n}-\Delta_{x}^{-} w_{j}^{n}}{\Delta x^{2}} \Delta x \\
& \leq \sup _{j, n}\left|v_{j}^{n}-v(j \Delta x, n \Delta t)\right|\left[\sum_{j \in S^{\prime}}\left(\left|\frac{\Delta_{x}^{2} v_{j}^{n}}{\Delta x^{2}}\right|+\left|\frac{\Delta_{x}^{2} v(j \Delta x, n \Delta t)}{\Delta x^{2}}\right|\right) \Delta x\right. \\
&\left.+2\left(C_{1}+\|v\|_{W^{1, \infty}}\right)\right],
\end{aligned}
$$

where we have used (1.2) and the definition of $w_{j}^{n}$. Now, from (1.3) we deduce, for any $j \in \mathbb{Z}$ and $n=1, \ldots, N$,

$$
u_{j}^{n}-u_{j-1}^{n} \leq C_{2} \Delta x,
$$

and therefore, setting $z_{j}^{n}:=u_{j}^{n}-C_{2} j \Delta x$, we obtain

$$
z_{j+1}^{n} \leq z_{j}^{n}
$$

for any $j \in \mathbb{Z}$ and $n=1, \ldots, N$. Then we have the following inequality:

$$
\begin{aligned}
\sum_{j \in S^{\prime}}\left|\frac{\Delta_{x}^{2} v_{j}^{n}}{\Delta x^{2}}\right| \Delta x & =\sum_{j \in S^{\prime}}\left|u_{j}^{n}-u_{j-1}^{n}\right| \leq \sum_{j \in S^{\prime}}\left|z_{j}^{n}-z_{j-1}^{n}\right|+C_{2}\left(j_{m}-j_{1}\right) \Delta x \\
& =z_{j_{1}}^{n}-z_{j_{m}}^{n}+C_{2}\left(j_{m}-j_{1}\right) \Delta x \\
& =u_{j_{1}}^{n}-u_{j_{m}}^{n}+2 C_{2}\left(j_{m}-j_{1}\right) \Delta x \\
& \leq 2\left(C_{1}+C_{2}|I|\right) .
\end{aligned}
$$

In a similar way we can estimate the term $\sum_{j \in S^{\prime}}\left|\frac{\Delta_{x}^{2} v(j \Delta x, n \Delta t)}{\Delta x^{2}}\right| \Delta x$ by using Lemma 4.1.

Finally, setting

$$
C_{3}:=4\left(C_{1}+\|v\|_{W^{1, \infty}}\right)+2\left(C_{2}+K\right)|I|,
$$

we get

$$
I_{1}=\int_{I}\left|u_{\Delta}(x, t)-\tilde{u}_{\Delta}(x, t)\right|^{2} \mathrm{~d} x \leq C_{3} \sup _{j, n}\left|v_{j}^{n}-v(j \Delta x, n \Delta t)\right|
$$


Second step: the estimate of $I_{2}$. We continue to use the previous notations. Then

$$
\begin{aligned}
I_{2}= & \int_{I}\left|\tilde{u}_{\Delta}(x, t)-u(x, t)\right|^{2} \mathrm{~d} x \\
\leq & 2 \sum_{j \in S} \int_{(j-1 / 2) \Delta x}^{(j+1 / 2) \Delta x} \mid \frac{v((j+1) \Delta x, n \Delta t)-v(j \Delta x, n \Delta t)}{\Delta x} \\
& +2 \int_{I}\left|\frac{v(x+\Delta x, t)-v(x, t)}{\Delta x}\right|^{2} \mathrm{~d} x \\
= & 2\left(J_{1}+J_{2}\right) .
\end{aligned}
$$

It is easily seen, from the Rademacher theorem, that

$$
J_{2}=O(\Delta x) \text {. }
$$

To estimate the term $J_{1}$, we shall follow the first step of the proof. Set

$$
w(x, t):=v(j \Delta x, n \Delta t)-v(x, t) \quad \text { for }(x, t) \in I_{j, n} .
$$

Then

$$
\begin{aligned}
J_{1}= & \sum_{j \in S} \int_{(j-1 / 2) \Delta x}^{(j+1 / 2) \Delta x}\left|\frac{w(x+\Delta x, t)}{\Delta x}-\frac{w(x, t)}{\Delta x}\right|^{2} \mathrm{~d} x \\
= & -\sum_{j \in S^{\prime}} \int_{(j-1 / 2) \Delta x}^{(j+1 / 2) \Delta x} w(x, t) \frac{\Delta_{x}^{2} w(x, t)}{\Delta x^{2}} \mathrm{~d} x \\
& +\int_{\left(j_{m}+1 / 2\right) \Delta x}^{\left(j_{m}+3 / 2\right) \Delta x} w(x, t)\left(\frac{w(x, t)-w(x-\Delta x, t)}{\Delta x^{2}}\right) \mathrm{d} x \\
& -\int_{\left(j_{1}-1 / 2\right) \Delta x}^{\left(j_{1}+1 / 2\right) \Delta x} w(x, t)\left(\frac{w(x+\Delta x, t)-w(x, t)}{\Delta x^{2}}\right) \mathrm{d} x .
\end{aligned}
$$

Since $v \in W^{1, \infty}(\mathbb{R} \times(0, T))$, we have immediately

$$
|w(x, t)| \leq\|v\|_{W^{1, \infty}} \sqrt{\Delta x^{2}+\Delta t^{2}} .
$$

Therefore, by using again Lemma 4.1, we have the following inequality:

$$
\begin{aligned}
& J_{1} \leq\|v\|_{W^{1, \infty}} \sqrt{\Delta x^{2}+\Delta t^{2}}\left[\sum_{j \in S^{\prime}}\left|\frac{\Delta_{x}^{2} v(j \Delta x, n \Delta t)}{\Delta x^{2}}\right| \Delta x\right. \\
&\left.\quad+\sum_{j \in S^{\prime}} \int_{(j-1 / 2) \Delta x}^{(j+1 / 2) \Delta x}\left|\frac{\Delta_{x}^{2} v(x, t)}{\Delta x^{2}}\right| \mathrm{d} x+4\|v\|_{W^{1, \infty}}\right] \\
& \leq\|v\|_{W^{1, \infty}} \sqrt{\Delta x^{2}+\Delta t^{2}}\left[\sum_{j \in S^{\prime}} \int_{(j-1 / 2) \Delta x}^{(j+1 / 2) \Delta x}\left|\frac{\Delta_{x}^{2} v(x, t)}{\Delta x^{2}}\right| \mathrm{d} x\right. \\
&\left.+6\|v\|_{W^{1, \infty}}+2 K|I|\right] .
\end{aligned}
$$


To conclude the proof, we only have to estimate the sum in the last inequality. Define

$$
\hat{u}_{\Delta}(x, t):=\frac{v(x+\Delta x, t)-v(x, t)}{\Delta x} .
$$

By Lemma 4.1, we have for any $(x, t) \in \mathbb{R} \times(0, T)$,

$$
\hat{u}_{\Delta}(x+\Delta x, t)-K(x+\Delta x) \leq \hat{u}_{\Delta}(x, t)-K x .
$$

Consequently, we can easily show, using the above arguments, that

$$
\begin{aligned}
& \sum_{j \in S^{\prime}} \int_{(j-1 / 2) \Delta x}^{(j+1 / 2) \Delta x}\left|\frac{\hat{u}_{\Delta}(x, t)-\hat{u}_{\Delta}(x-\Delta x, t)}{\Delta x}\right| \mathrm{d} x \\
& \quad \leq \frac{1}{\Delta x}\left[\int_{\left(j_{1}+1 / 2\right) \Delta x}^{\left(j_{1}+3 / 2\right) \Delta x} \frac{\left(\hat{u}_{\Delta}(x-\Delta x, t)-K(x-\Delta x)\right) \mathrm{d} x}{} \quad-\int_{\left(j_{m}-1 / 2\right) \Delta x}^{\left(j_{m}+1 / 2\right) \Delta x}\left(\hat{u}_{\Delta}(x, t)-K x\right) \mathrm{d} x\right]+K|I| \\
& \quad \leq 2\|v\|_{W^{\prime}, \infty}+2 K|I| .
\end{aligned}
$$

We conclude that

$$
J_{1} \leq C_{4}\|v\|_{W^{1, \infty}} \sqrt{\Delta x^{2}+\Delta t^{2}},
$$

where

$$
C_{4}:=8\|v\|_{W^{1, \infty}}+4 K|I| .
$$

The assertion follows from (4.4), (4.5), and (4.6).

We remark that the inequality (1.3) is a discrete analogue of the celebrated Oleinik entropy condition [23].

Remark 4.1. The proof of Theorem 1.1, for simplicity, has been given using a forward finite difference representation for $v_{x}$. The same result (and the same proof) holds true for centered or backward differences.

Remark 4.2. For compact support initial data for (CL), the condition (1.3) implies (1.2). In fact, we have the following

Proposition 4.2. Let $\left\{v_{j}\right\}$ be a real-valued sequence, $j \in \mathbb{Z}$, and $M, R, h_{0}, K$ some positive constants such that:

(a) for $|h j| \geq R$, for all $h \in\left(0, h_{0}\right)$ and $j \in \mathbb{Z}$

$$
v_{j+1}=v_{j}
$$

(b) for all $h \in\left(0, h_{0}\right)$ and $j \in \mathbb{Z}$

$$
\frac{v_{j+1}-2 v_{j}+v_{j-1}}{h^{2}} \leq K .
$$

Then, for all $h \in\left(0, h_{0}\right), j \in \mathbb{Z}$, we have

Proof. Set, for any $j \in \mathbb{Z}$,

$$
\left|\frac{v_{j+1}-v_{j}}{h}\right| \leq K\left(2 R+h_{0}\right) .
$$

$$
w_{j}=v_{j}-\frac{1}{2} K(h j)^{2}
$$


Then, by calculation,

$$
w_{j+1}-w_{j} \leq w_{j}-w_{j-1}, \quad \forall j .
$$

By applying (4.7) iteratively we can determine an upper bound for $w_{j+1}-w_{j}$ which is independent from $M$. In fact, for $|h j| \leq R$, we have

$$
\left|\frac{w_{j+1}-w_{j}}{h}\right| \leq \frac{1}{2} K\left(2 R+h_{0}\right),
$$

and therefore

$$
\left|\frac{v_{j+1}-v_{j}}{h}\right| \leq\left|\frac{w_{j+1}-w_{j}}{h}\right|+\frac{1}{2} K h(2 j+1) \leq K\left(2 R+h_{0}\right) .
$$

Remark 4.3. Let $I$ be any fixed interval of $\mathbb{R}$, and $S=\left\{j_{1}, \ldots, j_{m}\right\} \subseteq \mathbb{Z}$ be such that

$$
I \cap\left[\left(j_{i}-\frac{1}{2}\right) \Delta x,\left(j_{i}+\frac{1}{2}\right) \Delta x\right) \neq \varnothing
$$

for any $j_{i} \in S$. From (1.1) and (1.3) we have, for any $j \in \mathbb{Z}$ and $n=$ $1, \ldots, N$,

$$
u_{j}^{n}-u_{j-1}^{n} \leq C_{2} \Delta x
$$

and therefore, setting $z_{j}^{n}:=u_{j}^{n}-C_{2} j \Delta x$,

$$
z_{j+1}^{n} \leq z_{j}^{n} .
$$

Consequently, we have the following inequality for the total variation of $u_{j}^{n}$ on the interval $I$ :

$$
\begin{aligned}
\operatorname{TV}_{I}\left(u^{n}\right) \leq \sum_{i=2}^{m}\left|u_{j_{i}}^{n}-u_{j_{i}-1}^{n}\right| & \leq \sum_{i=2}^{m}\left|z_{j_{i}}^{n}-z_{j_{i}-1}^{n}\right|+C_{2}\left(j_{m}-j_{1}\right) \Delta x \\
& =z_{j_{1}}^{n}-z_{j_{m}}^{n}+C_{2}\left(j_{m}-j_{1}\right) \Delta x \\
& =u_{j_{1}}^{n}-u_{j_{m}}^{n}+2 C_{2}\left(j_{m}-j_{1}\right) \Delta x \\
& \leq 2\left(C_{1}+C_{2}|I|\right) .
\end{aligned}
$$

Thus, our schemes are locally TVB (i.e., they have bounded total variation). Nevertheless, we cannot use Harten's theorem [13] since, in general, they are not in conservation form (see also Proposition 5.3 and the Remark 5.1).

\section{CL-SCHEMES DERIVED FROM HJ-SCHEMES}

As we mentioned in the introduction, we can construct schemes satisfying the assumptions of the general convergence theorem, starting from the schemes which have been studied to obtain an approximation of the value function of a finite horizon optimal control problem.

We briefly describe here the origin of these methods referring to [11] and [10] for details.

Consider a system of controlled ordinary differential equations

$$
\left\{\begin{array}{l}
\dot{y}(s)=b(y(s), s, \alpha(s)), \quad s \in(t, T), \\
y(t)=x,
\end{array}\right.
$$


where $\alpha(\cdot) \in \mathscr{A}:=\{\alpha(\cdot):[0, T] \rightarrow A$, measurable $\}$ is the control, $A$ is a given subset of $\mathbb{R}^{m}$ and $y \in \mathbb{R}^{n}$ the state. The cost functional related to the finite horizon problem is

$$
\begin{aligned}
J(x, t, \alpha(\cdot)):= & \int_{t}^{T} g(y(s), s, \alpha(s)) e^{-\lambda(s-t)} \mathrm{d} s \\
& +\psi(y(T)) e^{-\lambda(T-t)},
\end{aligned}
$$

where $\lambda$ is a positive real parameter.

The value function is defined as

$$
w(x, t):=\inf _{\alpha(\cdot) \in \mathscr{A}} J(x, t, \alpha(\cdot)) .
$$

It is well known that, via the Dynamic Programming Principle, one can prove that $w$ satisfies the Hamilton-Jacobi-Bellman equation (HJB)

$$
\left\{\begin{array}{l}
-\frac{\partial}{\partial t} w+\lambda w+\sup _{a \in A}\left\{-b(x, t, a) \cdot \nabla_{x} w-g(x, t, a)\right\}=0 \text { in } \mathbb{R}^{n} \times(0, T), \\
w(x, T)=\psi(x) \text { in } \mathbb{R}^{n} .
\end{array}\right.
$$

Moreover (see, e.g., $[21, \S 8.4]), w$ is the unique viscosity solution of (HJB).

The scheme for the approximation of the value function $w$ is based on a discretization in time (step $k:=\Delta t$ ) of the control problem (5.1), (5.2). A Discrete Dynamic Programming Principle leads to a discrete (in time) version of (HJB). Adding a grid in the space variable (step $h:=\Delta x)$, one can get an approximation scheme ([11]) which, in the one-dimensional case, is

$$
(\mathrm{HJB})_{h}^{k} \begin{cases}w(j h, N k)=\psi(j h), & +k, g(j h, n k, a)\},\end{cases}
$$

where $n=0, \ldots, N$ and $N k=T$.

With $w_{j}^{n}$ denoting the approximate solution obtained by applying the above scheme, the following estimate holds (see [11]):

$$
\left|w(j h, n k)-w_{j}^{n}\right| \leq C_{1} k^{\frac{1}{2}}+C_{2} h+C_{3} \frac{h}{k} .
$$

We now show how this scheme can be applied to solve (HJ). We start recalling that whenever $f$ is a convex function, (HJ) can be written as

$$
\begin{cases}v_{t}+\sup _{a \in \mathbb{R}}\left\{a v_{x}-f^{*}(a)\right\}=0 & \text { in } \mathbb{R} \times(0, T), \\ v(x, 0)=v_{0}(x) & \text { in } \mathbb{R},\end{cases}
$$

where $f^{*}$ denotes the Legendre transform of $f$, that is,

$$
f^{*}(x)=\sup _{a \in \mathbb{R}}\{a x-f(a)\} \text { for any } x \in \mathbb{R} .
$$

By applying to (5.4) the change of variable

$$
v(x, t)=w(x, T-t) e^{\lambda t},
$$


we transform the original Cauchy problem for $v$ in the following problem with a terminal condition:

$$
\left\{\begin{array}{l}
-w_{t}+\lambda w+\sup _{a \in \mathbb{R}}\left\{a w_{x}-f^{*}(a) e^{-\lambda(T-t)}\right\}=0 \text { in } \mathbb{R} \times(0, T), \\
w(x, T)=v_{0}(x) \text { in } \mathbb{R},
\end{array}\right.
$$

i.e., we get the (HJB) corresponding to the control problem with $b(x, t, a) \equiv$ $-a$ and

$$
g(x, t, a) \equiv f^{*}(a) e^{-\lambda(T-t)} .
$$

In order to have a scheme for $(\mathrm{HJ})$, we do now the same, but in the opposite way, for the discrete problem. Then, introducing the change of variable

$$
w(j h, n k):=v(j h, T-n k) e^{-\lambda(T-n k)},
$$

we transform ( $\left.\mathrm{HJB}_{h}^{k}\right)$ into the following scheme for the Cauchy problem:

$$
\left\{\begin{aligned}
v(j h, n k)=\inf _{a \in \mathbb{R}}\{v(j h+k b(j h, T- & n k, a),(n-1) k) \\
& \left.+k g(j h, T-n k, a) e^{\lambda n k}\right\}, \\
v(j h, 0)=\psi(j h), &
\end{aligned}\right.
$$

which, in the special case of problem (5.4), corresponds to

$$
\left\{\begin{array}{l}
v_{j}^{n}=\inf _{a \in \mathbb{R}}\left\{v^{n-1}(j h-k a)+k f^{*}(a)\right\}, \\
v_{j}^{0}=v_{0}(j h),
\end{array}\right.
$$

where $v^{n}(x):=v(x, n k)$. To recover the information on the approximate solution of (CL) we just use (1.1) or any other discrete representation of $v_{x}$ (see Remark 4.1).

However, there are two main difficulties for computing the solution by (5.7). The first is related to the evaluation of the infimum, since we have to solve an extremum problem over an unbounded domain (notice that we are looking for the global minimum) and $f^{*}$ may be hard to compute. An algorithm for the computation of the Legendre transform has been proposed in [3], and precise error estimates (in $L^{\infty}$ ) have been established in [5].

The second difficulty is related to the observation that (5.7) is not a standard finite difference scheme, since in the right term appears $v^{n-1}(j h-k a)$, that is, $v^{n-1}$ computed on points which in general are not grid points. If this is the case, we replace $v^{n-1}(j h-k a)$ with the convex combination of $v_{q}^{n-1}$ and $v_{q+1}^{n-1}$, where $q \in \mathbb{Z}$ is such that $(j h-k a) \in[q h,(q+1) h]$.

Let us look more closely into the problem of computing the minimum in (5.7). Notice that, when $f \in C^{2}(\mathbb{R})$ is strictly convex, we can find an analytic expression for the convex function $f^{*}$. In fact, under these assumptions, for any $x \in \mathbb{R}$ there will be a unique $a$ such that $x=f^{\prime}(a)$. Since the supremum in (5.5) is achieved for $a=\left(f^{\prime}\right)^{-1}(x)$, the Legendre transform can be written as

$$
f^{*}(x)=x\left(f^{\prime}\right)^{-1}(x)-f\left(\left(f^{\prime}\right)^{-1}(x)\right) .
$$

Another useful property of the Legendre transform is the following: let $f$ be such that

$$
\lim _{|v| \rightarrow+\infty} \frac{f(v)}{|v|}=+\infty
$$

then $f^{*}$ satisfies (5.8) too, [14]. 
The following proposition shows that, if (5.8) holds, then the search for the supremum can be done over a bounded set.

Proposition 5.1. Assume that $v \in W^{1, \infty}(\mathbb{R})$ for any $t>0$. If $f$ is strictly convex and satisfies (5.8), there exists a bounded interval $A:=[-M, M]$, $M>0$, such that

$$
\sup _{a \in \mathbf{R}}\left\{a v_{x}-f^{*}(a)\right\}=\sup _{a \in A}\left\{a v_{x}-f^{*}(a)\right\} .
$$

Proof. Clearly, to get the result, it suffices to prove that there exists a constant $K$ such that the set

$$
A_{K}:=\left\{a \in \mathbb{R}: a v_{x}-f^{*}(a) \geq K\right\}
$$

is bounded and not empty. For any $a \in A_{K}$, we have

$$
\frac{f^{*}(a)+K}{|a|} \leq\left|v_{x}\right| \text {. }
$$

Since (5.8) holds and $\left\|v_{x}\right\| \leq \bar{V}$, the set $A_{K}$ is bounded for any $K$. To obtain $A_{K} \neq \varnothing$, we can choose

$$
K=-|\bar{a}| \bar{V}-f^{*}(\bar{a}),
$$

where $\bar{a}$ is any fixed value. In particular, (5.9) holds for the set

$$
A:=\left\{a \in \mathbb{R}:-|a| \bar{V}-f^{*}(a) \geq-f^{*}(0)\right\} .
$$

Notice also that (5.9) holds only if we assume that $f$ is Lipschitz continuous. In fact, by definition, in that case, $f^{*}(x)=+\infty$ for all $x,|x|>L_{f}$. Moreover, when (5.9) holds, if we let $h(x):=\arg \sup _{a \in A}\left\{a x-f^{*}(a)\right\}$, the argument associated with $x \in\left[-\left\|v_{0}\right\|_{W^{1, \infty}},\left\|v_{0}\right\|_{W^{1, \infty}}\right]$, then at least formally

$$
\frac{d}{d a}\left\{a x-f^{*}(a)\right\}_{a=h(x)}=0 \text {, }
$$

i.e.,

$$
\left(f^{*}\right)^{\prime}(h(x))=x
$$

Since

$$
f(x)=h(x) x-f^{*}(h(x)),
$$

differentiating and using $(5.10)$, we obtain

$$
f^{\prime}(x)=h(x)+h^{\prime}(x) x-\left(f^{*}\right)^{\prime}(h(x)) h^{\prime}(x)=h(x)
$$

and in particular $f^{\prime}\left(v_{x}\right)=h\left(v_{x}\right)$. The last implies that $A \supseteq[-R, R]$, where

$$
R=\sup _{x \in\left[-\left\|v_{0}\right\|_{W^{1}, \infty},\left\|v_{0}\right\|_{W^{1}, \infty}\right]}\left|f^{\prime}(x)\right| \text {. }
$$

As an example, consider the case $f(v):=\frac{1}{2} v^{2}$, which corresponds to the Burgers equation. It is straightforward to show that

$$
f^{*}(v)=\frac{1}{2} v^{2}
$$

and the supremum in (5.9) is achieved in the interval $\left[-\left\|v_{0}\right\|_{W^{1, \infty}},\left\|v_{0}\right\|_{W^{1, \infty}}\right]$. 
Remark 5.1: Representation formula for a nonconvex flux and approximation. Let $f$ be locally Lipschitz continuous and $v_{0}$ be Lipschitz continuous and convex; by $L_{v_{0}}$ and $L_{f}$ we will denote respectively their Lipschitz constants. Then the following representation formula for the solution of $(\mathrm{HJ})$ holds (see $[14,1])$ :

$$
v(x, t)=\sup _{y \in \mathbb{R}} \inf _{z \in \mathbb{R}}\left\{v_{0}(z)+y \cdot(x-z)-t f(y)\right\} .
$$

By the definition of the Legendre transform it is easy to prove that (5.11) can also be written as

$$
v(x, t)=\left(v_{0}^{*}+t f\right)^{*}(x) .
$$

Under the above assumptions the search for max and min can be restricted to two bounded closed intervals ([5]), $A \subseteq\{y: y \in \bar{B}(0, L)\}$ and $B=\{z: z \in$ $\left.\bar{B}\left(x, L_{f} t\right)\right\}$, so that

$$
v(x, t)=\max _{y \in A} \min _{z \in B}\left\{v_{0}(z)+y \cdot(x-z)-t f(y)\right\} .
$$

Then we can apply twice the algorithm studied in $[3,5]$. We remark that the representation formula (5.12) also suggests an approximation scheme which can be interpreted in terms of differential games. Let $x=j h, t=n k$, and $z=j h-k a$; substituting in (5.12), we have

$$
v_{j}^{n}=\max _{y \in A} \min _{|a| \leq L_{f}}\left\{v^{n-1}(j h-k a)+y k a-k f(y)\right\} .
$$

An algorithm for similar problems related to pursuit-evasion games has been studied in [2].

Now we want to prove that the HJ-scheme (5.7) satisfies the assumptions of the general convergence theorem when (5.9) is true. Let $a \in[-M, M]$; the point

if and only if

$$
(j h-k a) \in[(j+i) h,(j+i+1) h)=: I_{j i}
$$

$$
a \in\left(-(i+1) \frac{h}{k},-i \frac{h}{k}\right) \cap[-M, M]=: A_{i},
$$

where $p:=\left[\frac{k}{h} M\right]+1$ and $i \in P:=\{-p, \ldots, p-1\}$. Then the scheme can be written as

$$
v_{j}^{n+1}=\min _{i \in P}\left\{v_{j, i}^{n+1}\right\}
$$

where

$$
v_{j, i}^{n+1}:=\min _{a \in A_{i}}\left\{\left(1+i+\frac{k}{h} a\right) v_{j+i}^{n}-\left(i+\frac{k}{h} a\right) v_{j+i+1}^{n}+k f^{*}(a)\right\} .
$$

Theorem 5.2. Assume that $v_{0}$ satisfies (1.2), (1.3), and let (5.9) be true. Then the scheme (5.14), (5.15) satisfies assumptions (i) and (ii) of Theorem 1.1.

Proof. We first show that the scheme satisfies (i).

In fact, let

$$
v_{j+1}^{n}=v_{j+1, i_{1}}^{n}, \quad \text { where } i_{1} \in P,
$$


and

We have

$$
v_{j}^{n}=v_{j, i_{2}}^{n}, \quad \text { where } i_{2} \in P
$$

$$
v_{j+1}^{n}-v_{j}^{n}=v_{j+1, i_{1}}^{n}-v_{j, i_{2}}^{n} \leq v_{j+1, i_{2}}^{n}-v_{j, i_{2}}^{n} .
$$

Assume $a_{1}$ and $a_{2}$ are the points in $\left(-\left(i_{2}+1\right) \frac{h}{k},-i_{2} \frac{h}{k}\right] \cap[-M, M]$, where the minimum in (5.15) is achieved, respectively for $v_{j+1, i_{2}}^{n}$ and $v_{j, i_{2}}^{n}$. Then (5.16) becomes

$$
\begin{aligned}
v_{j+1}^{n}-v_{j}^{n} \leq & \left(1+i_{2}+\frac{k}{h} a_{1}\right) v_{j+1+i_{2}}^{n-1}-\left(i_{2}+\frac{k}{h} a_{1}\right) v_{j+1+i_{2}+1}^{n-1}+k f^{*}\left(a_{1}\right) \\
& -\left(1+i_{2}+\frac{k}{h} a_{2}\right) v_{j+i_{2}}^{n-1}+\left(i_{2}+\frac{k}{h} a_{2}\right) v_{j+i_{2}+1}^{n-1}-k f^{*}\left(a_{2}\right) \\
\leq & \left(1+i_{2}+\frac{k}{h} a_{2}\right)\left(v_{j+1+i_{2}}^{n-1}-v_{j+i_{2}}^{n-1}\right) \\
& -\left(i_{2}+\frac{k}{h} a_{2}\right)\left(v_{j+1+i_{2}+1}^{n-1}-v_{j+i_{2}+1}^{n-1}\right) \\
\leq & \max \left\{\left(v_{j+1+i_{2}}^{n-1}-v_{j+i_{2}}^{n-1}\right),\left(v_{j+2+i_{2}}^{n-1}-v_{j+i_{2}+1}^{n-1}\right)\right\}
\end{aligned}
$$

In the same way we get

$$
v_{j+1}^{n}-v_{j}^{n} \geq \min \left\{\left(v_{j+1+i_{1}}^{n-1}-v_{j+i_{1}}^{n-1}\right),\left(v_{j+2+i_{1}}^{n-1}-v_{j+i_{1}+1}^{n-1}\right)\right\},
$$

and (i) is proved by induction on the initial vector $\left\{v_{j}^{0}\right\}_{j \in \mathbf{Z}}$.

We now prove (ii). Set

$$
\begin{aligned}
v_{j+1}^{n} & =v_{j+1, i_{1}}^{n}, & & \text { where } i_{1} \in P, \\
v_{j}^{n} & =v_{j, i_{2}}^{n}, & & \text { where } i_{2} \in P, \\
v_{j-1}^{n} & =v_{j-1, i_{3}}^{n}, & & \text { where } i_{3} \in P .
\end{aligned}
$$

Then

$$
\begin{aligned}
v_{j+1}^{n}-2 v_{j}^{n}+v_{j-1}^{n} & =v_{j+1, i_{1}}^{n}-2 v_{j, i_{2}}^{n}+v_{j-1, i_{3}}^{n} \\
& \leq v_{j+1, i_{2}}^{n}-2 v_{j, i_{2}}^{n}+v_{j-1, i_{2}}^{n} .
\end{aligned}
$$

With $a_{1}, a_{2}$, and $a_{3}$ chosen as before, the above inequality becomes

$$
\begin{aligned}
v_{j+1}^{n}-2 v_{j}^{n}+v_{j-1}^{n} \leq & \left(1+i_{2}+\frac{k}{h} a_{2}\right) v_{j+1+i_{2}}^{n-1}-\left(i_{2}+\frac{k}{h} a_{2}\right) v_{j+i_{2}+2}^{n-1} \\
& -2\left(1+i_{2}+\frac{k}{h} a_{2}\right) v_{j+i_{2}}^{n-1}+2\left(i_{2}+\frac{k}{h} a_{2}\right) v_{j+i_{2}+1}^{n-1} \\
& +\left(1+i_{2}+\frac{k}{h} a_{2}\right) v_{j-1+i_{2}}^{n-1}-\left(i_{2}+\frac{k}{h} a_{2}\right) v_{j+i_{2}}^{n-1} \\
= & \left(1+i_{2}+\frac{k}{h} a_{2}\right)\left(v_{j+1+i_{2}}^{n-1}-2 v_{j+i_{2}}^{n-1}+v_{j+i_{2}-1}^{n-1}\right) \\
& -\left(i_{2}+\frac{k}{h} a_{2}\right)\left(v_{j+i_{2}+2}^{n-1}-2 v_{j+i_{2}+1}^{n-1}+v_{j+i_{2}}^{n-1}\right) \\
\leq & \max \left\{\left(v_{j+i_{2}+1}^{n-1}-2 v_{j+i_{2}}^{n-1}+v_{j+i_{2}-1}^{n-1}\right),\left(v_{j+i_{2}+2}^{n-1}-2 v_{j+i_{2}}^{n-1}+v_{j+i_{2}}^{n-1}\right)\right\} .
\end{aligned}
$$


Again, (ii) is proved by induction on the initial vector $\left\{v_{j}^{0}\right\}_{j \in \mathbf{Z}}$.

Theorem 5.2, by Theorem 1.1, gives a convergence result when the HJschemes converge, in particular, using (5.3), when $h=o(k)$.

We consider now the particular case when $\frac{k}{h} M<1$.

Proposition 5.3. Let $\frac{k}{h} M<1,(5.9)$ be true and $A=:[-M, M]$. Then the $C L$ scheme derived from the HJ-scheme (5.7) is a TVB, conservative and consistent three-point scheme; it is also consistent with the entropy inequality. Furthermore, if the CFL-number $\lambda:=\frac{k}{h}$ is small, the scheme is monotone.

Proof. Since $\frac{k}{h} M<1$ the CL-scheme is given by

$$
v_{j}^{n}=\min \left\{\bar{v}_{j}^{n} ; \underline{v}_{j}^{n}\right\},
$$

where

$$
\bar{v}_{j}^{n}:=\min _{a \in[0, M]}\left\{\left(1-\frac{k}{h} a\right) v_{j}^{n-1}+\frac{k}{h} a v_{j-1}^{n-1}+k f^{*}(a)\right\}
$$

and

$$
\underline{v}_{j}^{n}:=\min _{a \in[-M, 0]}\left\{\left(1+\frac{k}{h} a\right) v_{j}^{n-1}-\frac{k}{h} a v_{j+1}^{n-1}+k f^{*}(a)\right\} .
$$

If $v_{j}^{n}=\bar{v}_{j}^{n}$, then, by using $(1.1)$, we have

$$
v_{j}^{n}:=v_{j}^{n-1}+\min _{a \in[0, M]}\left\{-k a u_{j-1}^{n-1}+k f^{*}(a)\right\}
$$

So,

$$
\frac{v_{j}^{n}-v_{j}^{n-1}}{k}=\min _{a \in[0, M]}\left\{-a u_{j-1}^{n-1}+f^{*}(a)\right\} .
$$

In the same way, if $v_{j}^{n}=\underline{v}_{j}^{n}$, we have

$$
\frac{v_{j}^{n}-v_{j}^{n-1}}{k}=\min _{a \in[-M, 0]}\left\{-a u_{j}^{n-1}+f^{*}(a)\right\} \text {. }
$$

Since

$$
\begin{aligned}
u_{j}^{n} & =u_{j}^{n-1}+\frac{v_{j+1}^{n}-v_{j}^{n}}{h}-\frac{v_{j+1}^{n-1}-v_{j}^{n-1}}{h} \\
& =u_{j}^{n-1}+\frac{v_{j+1}^{n}-v_{j+1}^{n-1}}{h}-\frac{v_{j}^{n}-v_{j}^{n-1}}{h},
\end{aligned}
$$

from (5.17) and (5.18) we have the conservation form (3.1) if we define the numerical flux function to be

$$
\bar{f}_{j+\frac{1}{2}}^{n}:=\max \left\{\max _{a \in[0, M]}\left[a u_{j}^{n}-f^{*}(a)\right], \max _{a \in[-M, 0]}\left[a u_{j+1}^{n}-f^{*}(a)\right]\right\} .
$$

The consistency of the scheme, i.e., $f(u)=\bar{f}(u, \ldots, u)$ for any $u \in \mathbb{R}$, can be obtained simply using the definition of the Legendre transform. Moreover, the scheme is TVB from Remark 4.3 and the consistency with the entropy condition follows directly by (1.3). Finally, using the above definitions, we can easily prove that the function

$$
H\left(u_{j+1}^{n}, u_{j}^{n}, u_{j-1}^{n}\right):=u_{j}^{n}-\lambda\left[\bar{f}_{j+\frac{1}{2}}^{n}-\bar{f}_{j-\frac{1}{2}}^{n}\right]
$$


is monotone nondecreasing with respect to all its arguments, if $\lambda$ is small enough.

Remark 5.2: Conservation form and CFL condition for the HJ-schemes. Two different situations must be considered. The first is when $\frac{k}{h}$ is constant. Then the scheme is in conservation form and consistent but, in general, not monotone. Its convergence is guaranteed by Remark 4.3.

When $\frac{k}{h}$ is not constant, the CL-scheme obtained from the scheme (5.7) is not in conservation form, according to the classical Lax-Wendroff definition. In fact, the number of points involved in the scheme varies according to the ratio $k$. However, it is interesting to notice that the CFL condition is not violated by the scheme, since the domain of dependence of the finite difference method includes the domain of dependence of the differential operator. More precisely, if (5.9) holds, then for a fixed time step $k$ the domain of dependence at the grid point $x_{j}$ is given by the interval $\left[x_{j}-k M, x_{j}+k M\right]$ for any $h$. Now, by using (5.3), it is possible to increase the number of space grid points, without loss of stability. A numerical discussion of this point will be made in the following section (see, in particular, Tables 9 and 10).

Remark 5.3: Monotonicity for general HJ-schemes. In general, the monotonicity property will not hold for HJ-schemes. Consider the scheme (5.14), (5.15), so that the corresponding scheme for $(\mathrm{CL})$ can be written as

$$
u_{j}^{n+1}=\frac{v_{j+1}^{n+1}-v_{j}^{n+1}}{h}=\frac{1}{h}\left(\min _{i \in P} v_{j+1, i}^{n+1}-\min _{i \in P} v_{j, i}^{n+1}\right) .
$$

Assume that the the first minimum is obtained for $i=i_{1}$ and the second for $i=i_{2}$; by $(5.15)$ we get the following explicit scheme:

$$
\begin{aligned}
u_{j}^{n+1}= & \frac{v_{j+1+i_{1}}^{n}-v_{j+i_{2}}^{n}}{h}+\min _{a \in A_{i_{1}}}\left\{-\left(i_{1}+\frac{k}{h} a\right) u_{j+1+i_{1}}^{n}+\frac{k}{h} f^{*}(a)\right\} \\
& -\min _{a \in A_{i_{2}}}\left\{-\left(i_{2}+\frac{k}{h} a\right) u_{j+i_{2}}^{n}+\frac{k}{h} f^{*}(a)\right\} .
\end{aligned}
$$

If $i_{1}<i_{2}$, we will have

$$
\frac{v_{j+1+i_{1}}^{n}-v_{j+i_{2}}^{n}}{h}=-\sum_{s=0}^{i_{2}-i_{1}-2} u_{j+1+i_{1}+s}^{n} .
$$

Since all the terms $u_{j+1+i_{1}+s}^{n}, s=1, \ldots, j+i_{2}-1$, appear only in that sum, the scheme is not monotone with respect to these arguments.

Remark 5.4: Discretization of the Lax-Oleinik-Hopf representation formula. It is known $([19,23,14,21])$ that, when $f$ is convex and $x \in \mathbb{R}^{n}$, the solution of $(\mathrm{HJ})$ can be written as

$$
v(x, t)=\inf _{z \in \mathbb{R}^{n}}\left\{v_{0}(z)+t f^{*}\left(\frac{x-z}{t}\right)\right\} .
$$

As we have already noticed,

$$
z:=j h-k a \in I_{j i} \text { if and only if } a \in A_{i} .
$$


We also have

$$
z=\left(1+i+\frac{k}{h} a\right)(j+i) h-\left(i+\frac{k}{h} a\right)(j+i+1) h
$$

Moreover, we can always extend $v^{n}$ by linear interpolation on any interval $[j h,(j+1) h), j \in \mathbb{Z}$, i.e.,

$$
v^{n}(z):=\left(1+i+\frac{k}{h} a\right) v_{j+i}^{n}-\left(i+\frac{k}{h} a\right) v_{j+i+1}^{n} .
$$

Then, substituting in $(5.15)$, we have

$$
v_{j, i}^{n+1}=\min _{a \in A_{i}}\left\{v^{n}(z)+k f^{*}(a)\right\} .
$$

Recalling that $\bigcup_{i \in P} A_{i}=[-M, M]$ and that, under appropriate assumptions, the minimum for $a \in \mathbb{R}$ is achieved in a bounded interval $[-M, M]$, we can interprete the scheme (5.14), (5.15) as a discrete version of (5.21).

Remark 5.5: Local truncation error for the scheme related to the viscosity solution. Even if it seems difficult to derive a local truncation error for the general $\mathrm{HJ}$-scheme for (CL), owing to its rather involved formulation (see Remark 5.3), we can derive it for the viscosity solution.

Let $v$ be a sufficiently smooth solution of $(\mathrm{HJ})$ and $f$ be smooth. We set $x=j h, t=n k$, and we define the local truncation error for the scheme (5.7) (remember that in Proposition 5.1 we have already shown that the infimum is achieved on a compact set A) by

$$
L_{k}(x, t)=\frac{1}{k}\left[v(x, t)-\min _{a \in A}\left\{v(x-k a, t-k)+k f^{*}(a)\right\}\right] .
$$

Denoting the difference in the square brackets by $D_{k}(x, t)$ and developing by Taylor expansion, we get

$$
\begin{aligned}
D_{k}(x, t)= & v(x, t)-\min _{a \in A}\left\{v(x, t-k)+v_{x}(x, t-k)(-k a)\right. \\
= & \left.v(x, t)-v(x, t-k) \quad+v_{x x}(x, t-k)(k a)^{2}+\cdots+k f^{*}(a)\right\} \\
& \quad+k \max _{a \in A}\left\{v_{x}(x, t-k) a-v_{x x}(x, t-k) k a^{2}+\cdots+f^{*}(a)\right\} \\
= & v_{t}(x, t) k+\frac{1}{2} v_{t t}(x, t) k^{2}+O\left(k^{2}\right)+k f\left(v_{x}(x, t-k)\right) \\
= & v_{t}(x, t) k+\frac{1}{2} v_{t t}(x, t) k^{2}+O\left(k^{2}\right) \\
& +k\left[f\left(v_{x}(x, t)\right)+f^{\prime}\left(v_{x}(x, t)\right)\left(v_{x}(x, t-k)-v_{x}(x, t)\right)+\cdots\right] .
\end{aligned}
$$

Since $v$ is a solution of $(\mathrm{HJ})$, we can conclude that

$$
L_{k}(x, t)=\frac{1}{k}\left[\frac{1}{2} v_{t t}(x, t) k^{2}+O\left(k^{2}\right)+f^{\prime}\left(v_{x}(x, t)\right) v_{x t}(x, t) k^{2}+\cdots\right]=O(k) .
$$

\section{TESTS, EXAMPLES AND NUMERICAL EFFICIENCY}

The numerical results in this section deal with the solution of the Burgers equation $u_{t}+\left(\frac{1}{2} u^{2}\right)_{x}=0$. In this example, (5.7) is 


$$
v_{j}^{n}=\min _{a \in[-M, M]}\left\{v^{n-1}(j h-k a)+\frac{k}{2} a^{2}\right\},
$$

where $M$ is the Lipschitz constant of the initial data for the corresponding problem $(\mathrm{HJ})$.

As one can observe, the number of space nodes necessary to compute $v_{j}^{n}$ at each time step depends strictly on $h$ and $k$. If, for example,

$$
\frac{k}{h}|a| \leq 1 \quad \forall a \in[-M, M],
$$

the scheme is a three-point scheme and

$$
v_{j}^{n}=\min \left\{\bar{v}_{j}^{n} ; \underline{v}_{j}^{n}\right\},
$$

where

$$
\bar{v}_{j}^{n}:=\min _{a \in[0, M]}\left\{\left(1-\frac{k}{h} a\right) v_{j}^{n-1}+\frac{k}{h} a v_{j-1}^{n-1}+\frac{k}{2} a^{2}\right\}
$$

and

$$
\underline{v}_{j}^{n}:=\min _{a \in[-M, 0]}\left\{\left(1+\frac{k}{h} a\right) v_{j}^{n-1}-\frac{k}{h} a v_{j+1}^{n-1}+\frac{k}{2} a^{2}\right\} .
$$

A simple case study leads to the following explicit formulas:

$$
\begin{gathered}
\bar{v}_{j}^{n}=\left\{\begin{array}{rr}
v_{j}^{n-1} & \text { if } v_{j}^{n-1} \leq v_{j-1}^{n-1}, \\
v_{j}^{n-1}-\frac{k}{h^{2}}\left[\frac{1}{2}\left(v_{j}^{n-1}\right)^{2}+\frac{1}{2}\left(v_{j-1}^{n-1}\right)^{2}-v_{j-1}^{n-1} v_{j}^{n-1}\right] . & \text { if } v_{j-1}^{n-1}<v_{j}^{n-1} \leq v_{j-1}^{n-1}+M h, \\
v_{j}^{n-1}-\frac{k}{h} M\left[v_{j}^{n-1}-v_{j-1}^{n-1}\right]+\frac{k}{2} M^{2} & \text { if } v_{j-1}^{n-1}+M h<v_{j}^{n-1},
\end{array}\right. \\
\underline{v}_{j}^{n}=\left\{\begin{array}{rr}
v_{j}^{n-1} & \text { if } v_{j}^{n-1} \leq v_{j+1}^{n-1}, \\
v_{j}^{n-1}-\frac{k}{h^{2}}\left[\frac{1}{2}\left(v_{j}^{n-1}\right)^{2}+\frac{1}{2}\left(v_{j+1}^{n-1}\right)^{2}-v_{j+1}^{n-1} v_{j}^{n-1}\right] \\
v_{j}^{n-1}-\frac{k}{h} M\left[v_{j}^{n-1}-v_{j+1}^{n-1}\right]+\frac{k}{2} M^{2} & \text { if } v_{j+1}^{n-1}<v_{j}^{n-1} \leq v_{j+1}^{n-1}+M h, v_{j}^{n-1} .
\end{array}\right.
\end{gathered}
$$

In contrast, if we choose $h=k^{3 / 2}$ to respect (5.3) and get the estimate

$$
\left\|w-w_{j}^{n}\right\|_{L^{\infty}} \leq C k^{1 / 2},
$$

the number of points increases, the accuracy of the method increases too, and for the computation of the minimum we repeat the same arguments as before with more cases to be considered. For a general convex flux $f$, one should use (5.7) and an approximation of $f^{*}$ (e.g., by the algorithm described in $[3,6]$ ).

For the numerical experiments, we have chosen for (CL) three initial data $u_{0}$ such that the corresponding $v_{0}$ has Lipschitz constant $M=1$. In all figures the exact solution is represented by a continuous line, whereas the approximate solution is represented by small circles. All the tests have run on a VAX $6500 / 510$, and the CPU time reported in the tables (see Supplement) is in the 
form min:sec.dec. or sec.dec. As we have already remarked, one can use any discretization of $v_{x}$; the numerical results in all the tables refer to a centered difference scheme. In all the tests we have set the viscosity coefficient for the Harten scheme equal to 0.25 .

Test 1. The initial data is

$$
u_{0}(x)= \begin{cases}1 & \text { for } x \in[-1,1] \\ 0 & \text { elsewhere }\end{cases}
$$

and we have looked for the solution at time $T=2$. Using characteristics, one can easily prove that the exact solution has a shock at that time.

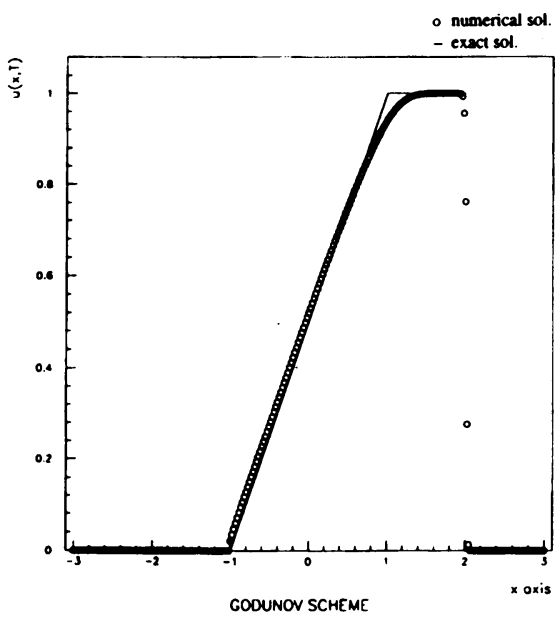

(a)

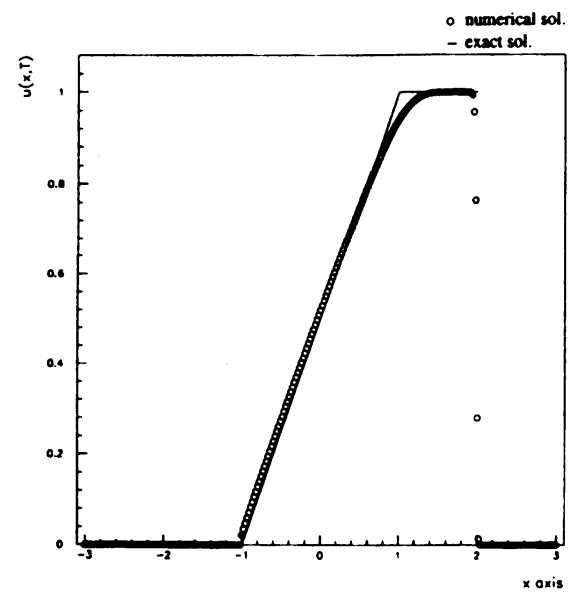

(c)

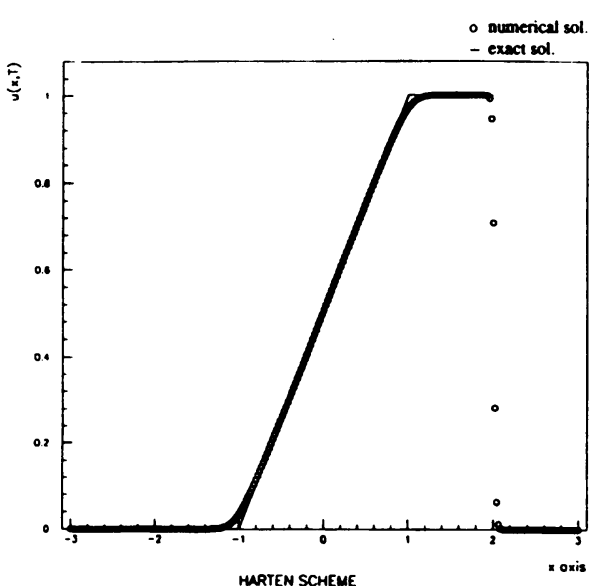

(b)

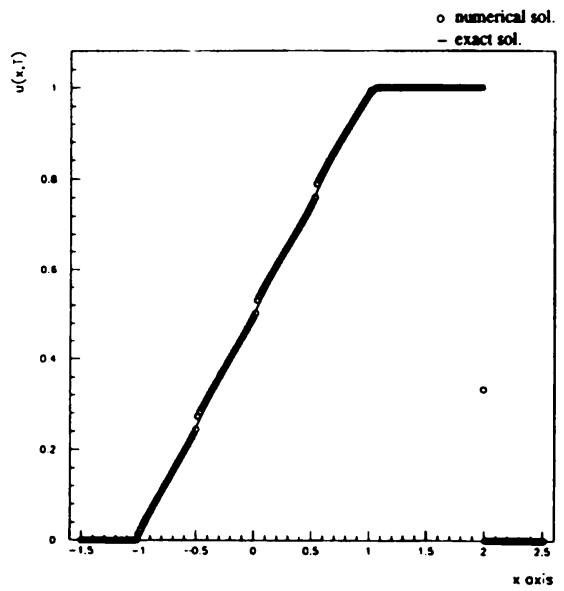

(d)

Figure 1. Test 1: exact versus approximate solutions at time $T=2$

(a) Godunov: space nodes $=270$, time nodes $=405$

(b) Harten: space nodes $=270$, time nodes $=405$

(c) H-J scheme $k \leq h$ : space nodes $=270$, time nodes $=405$

(d) H-J scheme $h=k^{3 / 2}$ : space nodes $=233$, time nodes $=30$ 
Figures $1 \mathrm{c}, \mathrm{d}$ show the numerical results for the three-point scheme, and for the general scheme where $h=k^{3 / 2}$. They are compared with the results obtained by applying the Godunov scheme and the Harten scheme [13] to the same problem (Figures 1a,b). The same number of nodes in space and time has been used for the three-point $\mathrm{HJ}$-scheme, Godunov and Harten methods, since for these methods a condition of type (6.2) is needed. It is rather interesting to compare the results; in particular, notice that $1 \mathrm{c}$ is almost equal to $1 \mathrm{a}$ and that the general HJ-scheme converges even better where the solution has a jump with roughly the same number of nodes in space and less steps in time (30 instead 405). In Figure 1d one can see a strange oscillation around the exact solution but the corners of the entropy solution are picked up very well, and the diffusion around the shock is almost negligible ( 1 node).

Table 1 contains the errors (in the $L^{1}$-norm) of our methods for different mesh sizes and time steps. This table confirms that, in this test, the general $\mathrm{HJ}$-scheme is more accurate and requires less CPU time.

In Table 2 we compare the orders of convergence in $L^{1}$ for all the above schemes. Notice that the general HJ scheme has a higher order of convergence ( 0.9 instead of 0.8$)$ and it is not very far from the order of convergence of Harten's scheme.

We have computed the approximate order of convergence $\gamma$ in terms of the space step $h$. Assuming for the error in the $L^{1}$-norm the estimate

$$
\operatorname{Err}(h)=O\left(h^{\gamma}\right),
$$

we have computed the error for $h_{1}$ and $h_{2}$ to obtain

$$
\frac{\operatorname{Err}\left(h_{1}\right)}{\operatorname{Err}\left(h_{2}\right)}=O\left(\frac{h_{1}}{h_{2}}\right)^{\gamma}
$$

Typically for the methods satisfying (6.2), we have chosen $h_{2}=h_{1} / 2$. The order for the HJ-schemes where $h=k^{\frac{3}{2}}$ has been computed always in terms of the space step $h$, choosing $k_{2}=k_{1} / 4$, so that $h_{2}=h_{1} / 8$.

Test 2. The initial data is

$$
u_{0}(x)= \begin{cases}1-x & \text { for } x \in[0,1] \\ 0 & \text { elsewhere }\end{cases}
$$

and we have computed the solution up to time $T=0.7$.

Again, we compare our methods with Godunov and Harten schemes in Figure 2. The general HJ-scheme behaves very well also in this test (see 2d), staying close to the sharp cusp, while the other methods smooth it out. Notice that the result of Figure $2 \mathrm{~d}$ has been obtained using only 20 nodes in time.

For this example we have computed the errors in $L^{1}$ and $L^{\infty}$; they are shown in Table 3. The difference between the errors in $L^{\infty}$ of Tables 3a and $3 \mathrm{~b}$ is very large but also the difference in terms of the $L^{1}$ error is substantial if we compare the errors which correspond to similar CPU times.

Finally, Table 4 shows the order of convergence in $L^{1}$ and $L^{\infty}$. In this test the general HJ-scheme has the best order of convergence (even better than the order of Harten's scheme). 


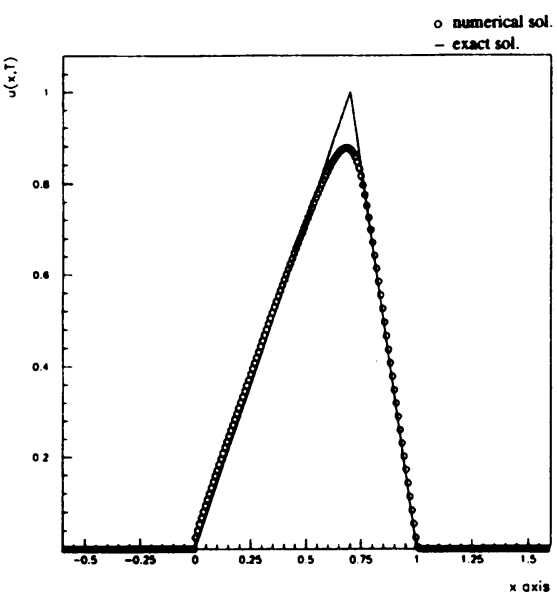

(a)

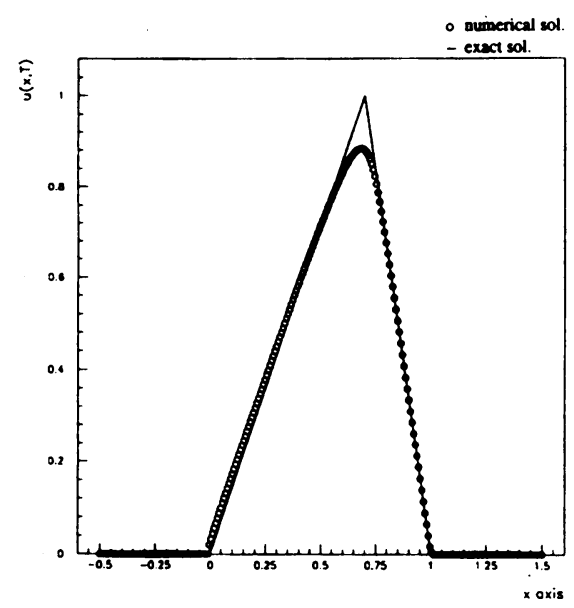

(c)

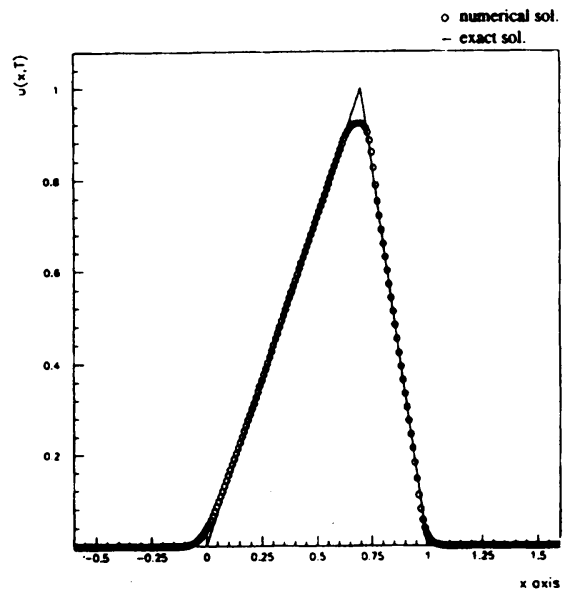

(b)

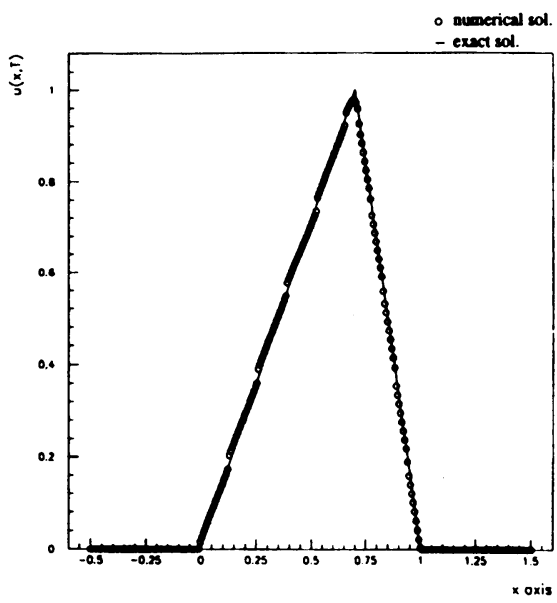

(d)

FIGURE 2. Test 2: exact versus approximate solutions at time $T=0.7$

(a) Godunov : space nodes $=270$, time nodes $=405$

(b) Harten: space nodes $=270$, time nodes $=405$

(c) H-J scheme $k \leq h$ : space nodes $=270$, time nodes $=405$

(d) H-J scheme $h=k^{3 / 2}$ : space nodes $=306$, time nodes $=20$

Test 3. This test shows the behavior of HJ-schemes when the solution is smooth. The initial data is $u_{0}(x)=0.5+\sin (\pi x)$ with periodic boundary conditions, and the solution is computed up to time $T=0.2$.

We have taken advantage of the periodic boundary conditions in the implementation of Godunov and Harten schemes. Notice that this cannot be done for the $\mathrm{HJ}$-schemes since the corresponding initial condition for $(\mathrm{HJ})$ is not periodic. 


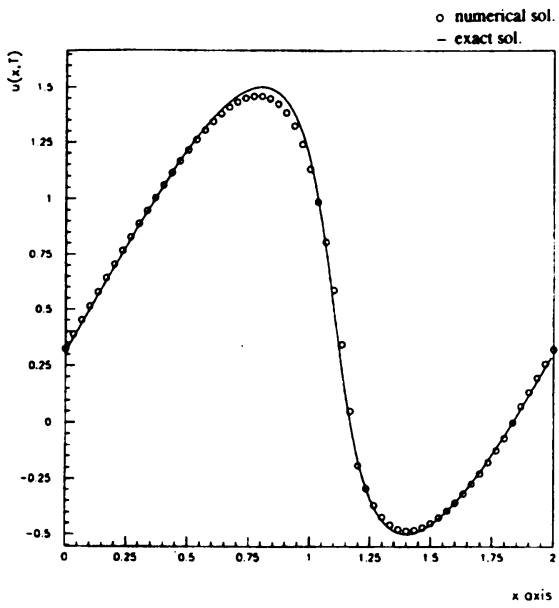

(a)

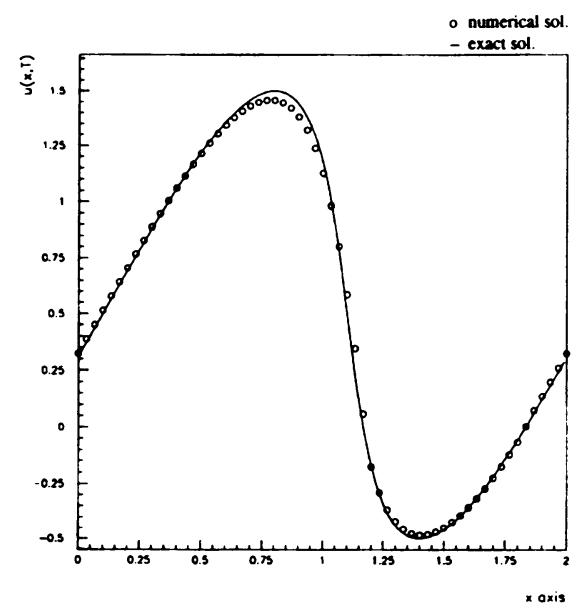

(c)

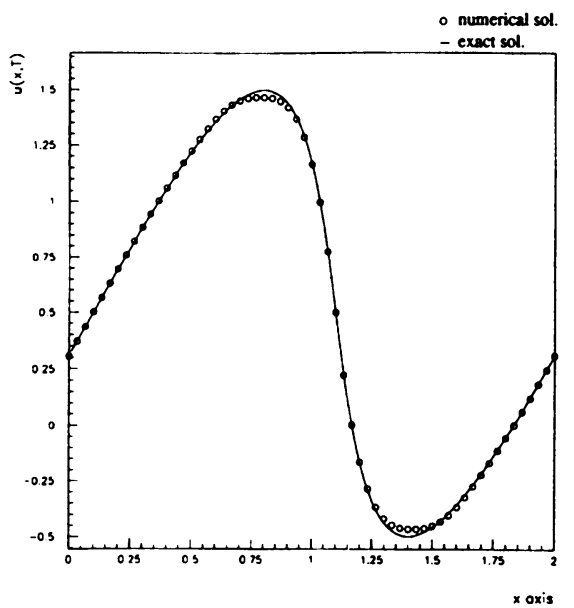

(b)

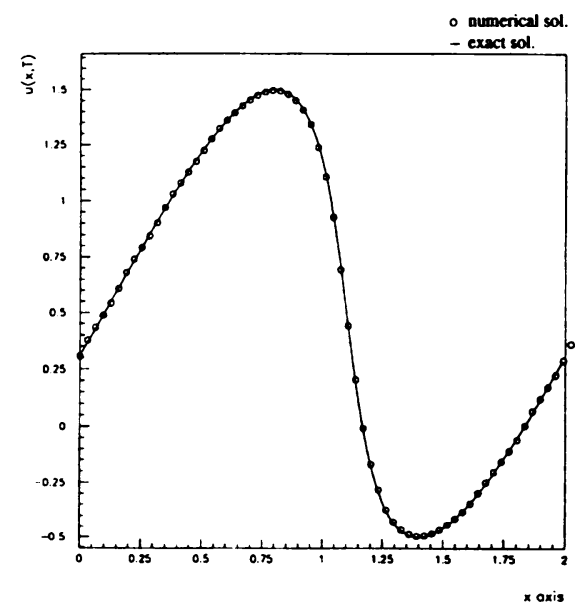

(d)

FIgURE 3. Test 3: exact versus approximate solutions at time $T=0.2$

(a) Godunov : space nodes $=60$, time nodes $=90$

(b) Harten : space nodes $=60$, time nodes $=90$

(c) H-J scheme $k \leq h$ : space nodes $=60$, time nodes $=90$

(d) H-J scheme $h=k^{3 / 2}$ : space nodes $=64$, time nodes $=2$

In Figure 3 we show the drawings corresponding to the above methods. The approximate solution corresponding to the general $\mathrm{HJ}$-scheme stays close to the solution also near the local maximum and minimum points (where the solutions obtained by the other methods differ from the exact solution). Notice that the result of Figure $3 \mathrm{~d}$ has been obtained using only 2 nodes in time (i.e., $k=0.1$ ).

Table 5 contains the errors in $L^{1}$ and $L^{\infty}$ and the CPU times corresponding to a number of different time steps and mesh sizes. In 02.28 seconds the general $\mathrm{HJ}$-scheme produces a solution with an $L^{\infty}$ error of 0.0154 . The Harten scheme 
uses 3.01 seconds to obtain a similar $L^{\infty}$ error and the other methods never reach that accuracy. In Table 6 we show the orders of convergence in $L^{1}$ and $L^{\infty}$. Table 9 contains the results for a $\mathrm{HJ}$-scheme where the choice of $h$ is independent from $k$. In fact, even if the a priori estimate (5.3) suggests that the choice $h=k^{3 / 2}$ is optimal, a different choice is still possible. The best result is obtained with only two steps in time and the smallest mesh size $(h=0.001)$. The orders of convergence are always close to, or above, 1.

Test 4. This test shows the behavior of HJ-schemes in smooth regions after a shock appears. We have considered the same initial data (with periodic boundary conditions) of Test 3 but the solution is computed up to $T=1$. As one can easily prove, the entropy solution has a jump. In Tables 7 and 8 we compare the methods in term of the errors, CPU time and order of convergence. In this example we have computed also the $L^{\infty}$ error related to the region where the solution is smooth (in particular, this error refers to the set $I \backslash I_{0}$, where $I_{0}=(\bar{x}-0.1, \bar{x}+0.1)$ and $\bar{x}$ is the point of discontinuity). Notice that HJ-schemes fit well the exact solution in the regularity region.

Tables 11 and 12 contain the results for an HJ-scheme where the choice of $h$ is independent of $k$. Also in this test, the best result is obtained with only two steps in time and the smallest mesh size. The approximate solution stays very close to the exact solution in the region of regularity (the corresponding $L^{\infty}$ error is about $10^{-2}$ or less). The orders of convergence are close to 1 .

Conclusion. The numerical results show that the HJ-schemes that we propose are very accurate and faster than our implementation of the methods of Godunov and Harten. We observe that the general HJ-scheme stays very close to the solution wherever it is continuous (in particular, look at the results for Test 2 ): in fact the main contribution to its $L^{1}$ error is given by the jump around the shocks (see Test 4). Even better results can be obtained for large time steps and small mesh sizes (see Tables 9 and 11).

Finally, we observe that, for the same $L^{1}$ error, the CPU times of the general $\mathrm{HJ}$-scheme are drastically lower than those of the other methods.

\section{ACKNOWLEDGMENTS}

We wish to thank P. Marcati for many helpful and enlightening discussions.

\section{BIBLIOGRAPHY}

1. M. Bardi and L. Evans, On Hopf's formulas for solutions of Hamilton-Jacobi equations, Nonlinear Anal. TMA 8 (1984), 1373-1381.

2. M. Bardi, M. Falcone, and P. Soravia, Fully discrete schemes for the value function of pursuit-evasion games, Advances in Dynamic Games and Applications (T. Basar and A. Haurie, eds.), Birkhäuser, 1994, pp. 89-105.

3. Y. Brenier, Un algorithme rapide pour le calcul de transformées de Legendre-Fenchel discrètes, C.R. Acad. Sci. Paris Ser. I 308 (1989), 587-589.

4. V. Caselles, Scalar conservation laws and Hamilton-Jacobi equations in one-space variables, Nonlinear Anal. TMA 18 (1992), 461-469.

5. L. Corrias, Transformées de Legendre-Frenchel discrètes et applications, Memoire de D.E.A., Laboratoire d'Analyse Numérique, Université Pierre et Marie Curie, 1992. 
6. M. G. Crandall, H. Ishi, and P. L. Lions, User's guide to viscosity solutions of second order partial differential equations, Bull. Amer. Math. Soc. (N.S.) 27 (1992), 1-67.

7. M. G. Crandall, L. Evans, and P. L. Lions, Some properties of viscosity solutions of Hamilton-Jacobi equations, Trans. Amer. Math. Soc. 282 (1984), 487-502.

8. M. G. Crandall and P. L. Lions, Viscosity solutions of Hamilton-Jacobi equations, Trans. Amer. Math. Soc. 277 (1983), 1-42.

9. - Two approximations of solutions of Hamilton-Jacobi equations, Math. Comp. $\mathbf{4 3}$ (1984), 1-19.

10. M. Falcone, A numerical approach to the infinite horizon problem, Appl. Math. Optim. 15 (1987), 1-13 and 23 (1991), 213-214.

11. M. Falcone and T. Giorgi, An approximation scheme for evolutive Hamilton-Jacobi equations, preprint, 1994.

12. A. Harten, On a large time-step high resolution scheme, Math. Comp. 46 (1986), 379-400.

13., High resolution schemes for hyperbolic conservation laws, J. Comput. Phys. 49 (1983), 357-393.

14. E. Hopf, Generalized solutions of non-linear equations of first order, J. Math. Mech. 14 (1965), 951-973.

15. S. N. Kružkov, First order quasilinear equations in independent variables, Mat. Sb. 81 (1970), 228-255; English transl. in Math. USSR-Sb. 10 (1970), 217-243.

16. - The Cauchy problem in the large for certain non-linear first order differential equations, Dokl. Akad. Nauk SSSR 132 (1960); English transl. in Soviet Math. Dokl. 1 (1960), 474-477.

17. _ The method of finite differences for a first-order non-linear equation with many independent variables, Zh. Vychisl. Mat. i Mat. Fiz. 5 (1966), 884-894.

18. __ The Cauchy problem in the large for nonlinear equations and for certain quasilinear systems of the first order with several variables, Dokl. Akad. Nauk SSSR 155 (1964); English transl. in Soviet Math. Dokl. 5 (1964), 493-496.

19. P. D. Lax, Hyperbolic systems of conservation laws and the mathematical theory of shock waves, SIAM Regional Conf. Series, Lectures in Applied Math., vol. 11, 1970, pp. 1-47.

20. R. J. LeVeque, A large time step generalization of Godunov's method for systems of conservation laws, SIAM J. Numer. Anal. 22 (1985), 1051-1073.

21. P. L. Lions, Generalized solutions of Hamilton-Jacobi equations, Pitman, London, 1982.

22. P. L. Lions and P. E. Souganidis, Convergence of MUSCL type methods for scalar conservation laws, C.R. Acad. Sci. Paris Ser. I 311 (1990), 259-264.

23. O. A. Oleinik, Uniqueness and stability of the generalized solution of the Cauchy problem for a quasi-linear equation, Uspekhi Mat. Nauk 14 (1959), 165-170; English transl. in Amer. Math. Soc. Transl. (2) 33 (1963), 285-290.

24. S. Osher, Riemann solvers, the entropy condition and difference approximation, SIAM J. Numer. Anal. 21 (1984), 217-235.

25. S. Osher and J. A. Sethian, Fronts propagating with curvature-dependent speed: Algorithms on Hamilton-Jacobi formulations, J. Comput. Phys. 79 (1988), 12-49.

(Corrias and Natalini) Istituto per le Applicazioni del Calcolo "M. Picone", Viale del Policlinico 137 - 00161 Rome, ItAly

E-mail address: natalini@vaxiac.iac.rm.cnr.it

(Falcone) Dipartimento di Matematica "G. Castelnuovo", Universita di Roma "La SAPIENZA", P.zZA A. MoRo 2 - 00185 Rome, ItAly

E-mail address: falcone@sci.uniroma1.it 


\section{Supplement to}

\section{NUMERICAL SCHEMES FOR CONSERVATION LAWS VIA HAMILTON-JACOBI EQUATIONS}

\section{CORRIAS, M. FALCONE, AND R. NATALINI}

Table 1. Test 1: maximum $L^{1}$ errors and CPU times.

(a) $(\mathrm{H})=$ Harten scheme, $(\mathrm{G})=$ Godunov scheme, $(\mathrm{HJ})=\mathrm{H}-\mathrm{J}$ scheme with $k \leq h$

(b) H-J scheme $h=k^{3 / 2}$

\begin{tabular}{|c|c|c|c|}
\hline Time Step & Mesh Size & $L^{1}$ Error & CPU \\
\hline$k=0.066666$ & $h=0.300000$ & $\begin{array}{ll}\text { (H) } & 0.446665 \\
\text { (G) } & 0.474162 \\
\text { (HJ) } & 0.526606\end{array}$ & $\begin{array}{ll}\text { (H) } & 0.75 \\
\text { (G) } & 0.68 \\
\text { (HJ) } & 0.63\end{array}$ \\
\hline$k=0.033333$ & $h=0.150000$ & $\begin{array}{ll}\text { (H) } & 0.226174 \\
\text { (G) } & 0.266013 \\
\text { (HJ) } & 0.313992\end{array}$ & $\begin{array}{ll}\text { (H) } & 1.07 \\
\text { (G) } & 0.69 \\
\text { (HJ) } & 0.58\end{array}$ \\
\hline$k=0.016666$ & $h=0.075000$ & $\begin{array}{ll}\text { (H) } & 0.116579 \\
\text { (G) } & 0.173682 \\
\text { (HJ) } & 0.177846\end{array}$ & $\begin{array}{ll}\text { (H) } & 2.38 \\
\text { (G) } & 0.83 \\
\text { (HJ) } & 0.78\end{array}$ \\
\hline$k=0.008333$ & $h=0.037500$ & $\begin{array}{ll}\text { (H) } & 0.056856 \\
\text { (G) } & 0.091962 \\
\text { (HJ) } & 0.103086\end{array}$ & $\begin{array}{ll}\text { (H) } & 6.40 \\
\text { (G) } & 1.22 \\
\text { (HJ) } & 1.13\end{array}$ \\
\hline
\end{tabular}

(a)

\begin{tabular}{|c|c|c|c|}
\hline Time Step & Mesh Size & $L^{1}$ Error & CPU \\
\hline$k=0.200$ & $h=0.089442$ & 0.154003 & 00.75 \\
$k=0.100$ & $h=0.031622$ & 0.055008 & 01.33 \\
$k=0.080$ & $h=0.022627$ & 0.040224 & 01.55 \\
$k=0.050$ & $h=0.011180$ & 0.021983 & 04.56 \\
$k=0.040$ & $h=0.008000$ & 0.014811 & 05.63 \\
$k=0.025$ & $h=0.003952$ & 0.008301 & 29.91 \\
$k=0.020$ & $h=0.002828$ & 0.006038 & 57.19 \\
\hline
\end{tabular}

(b) 

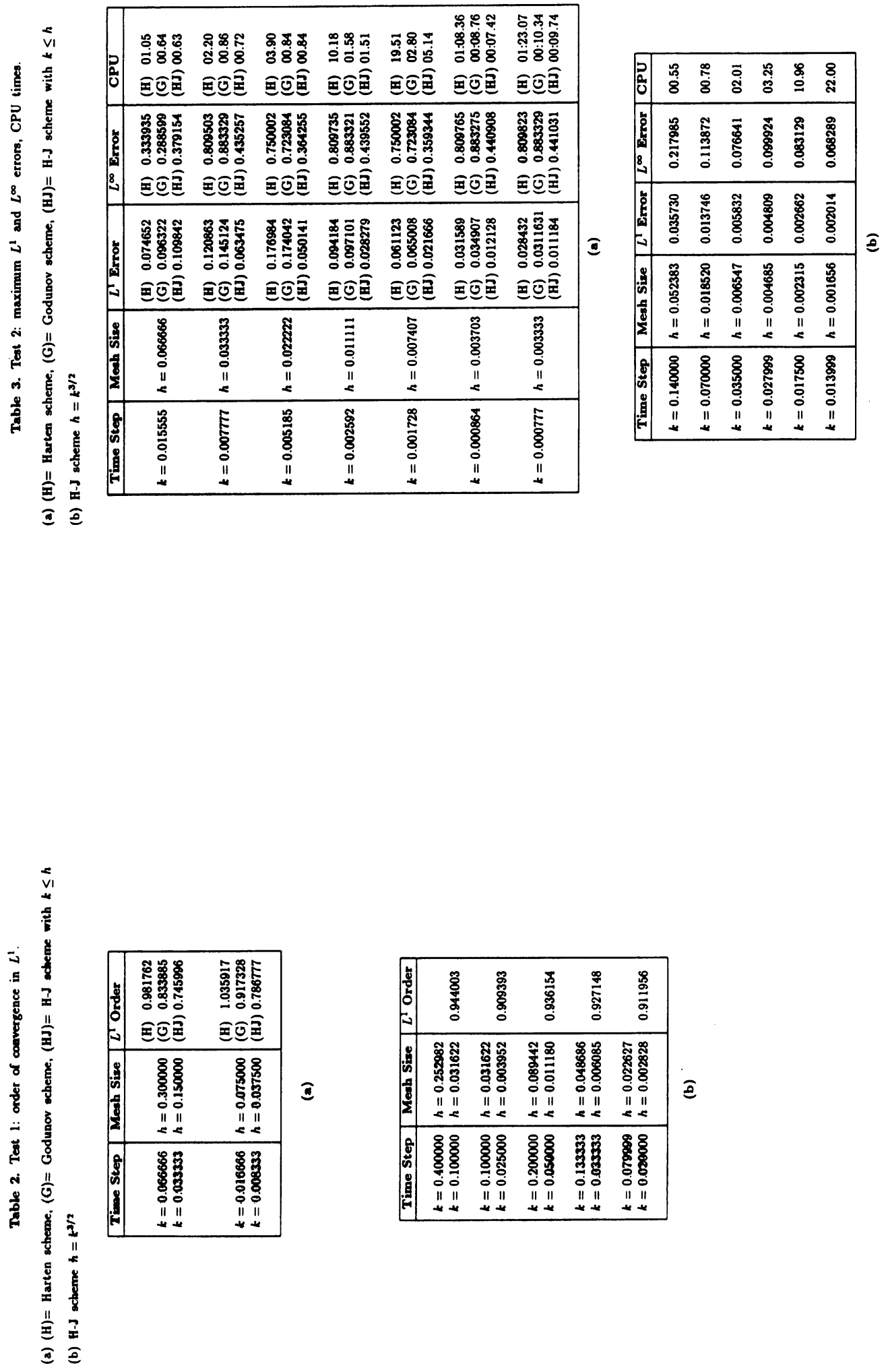

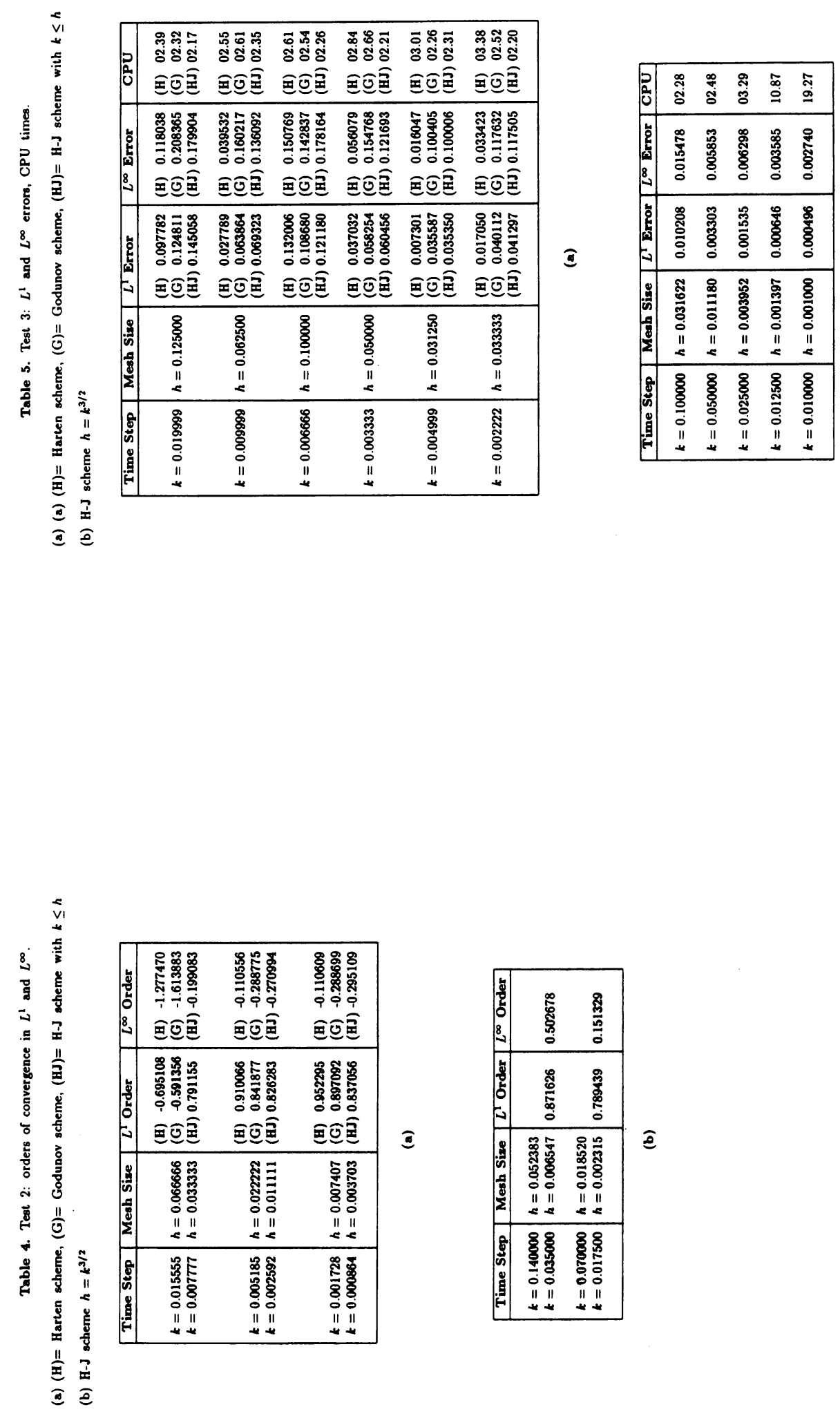

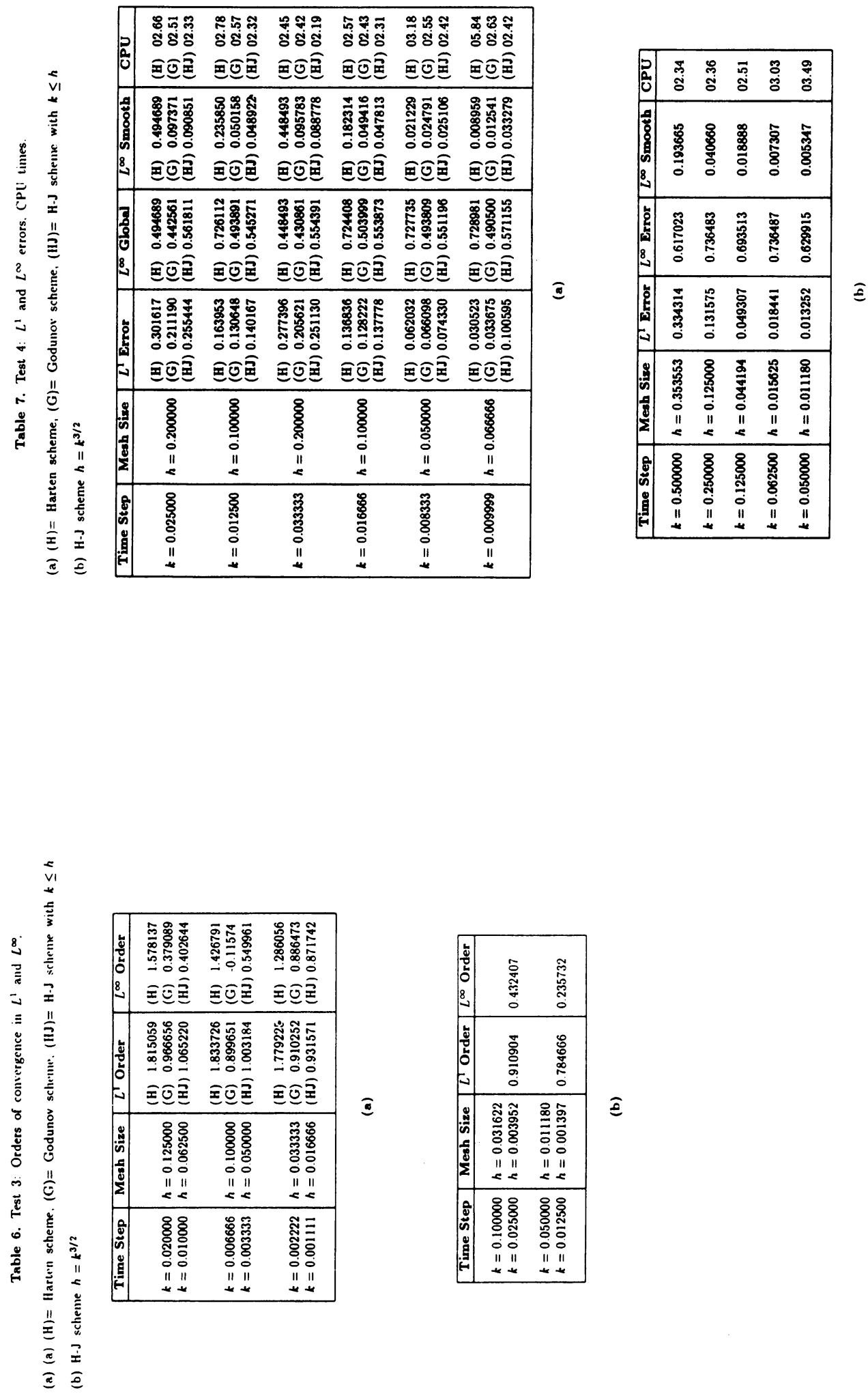

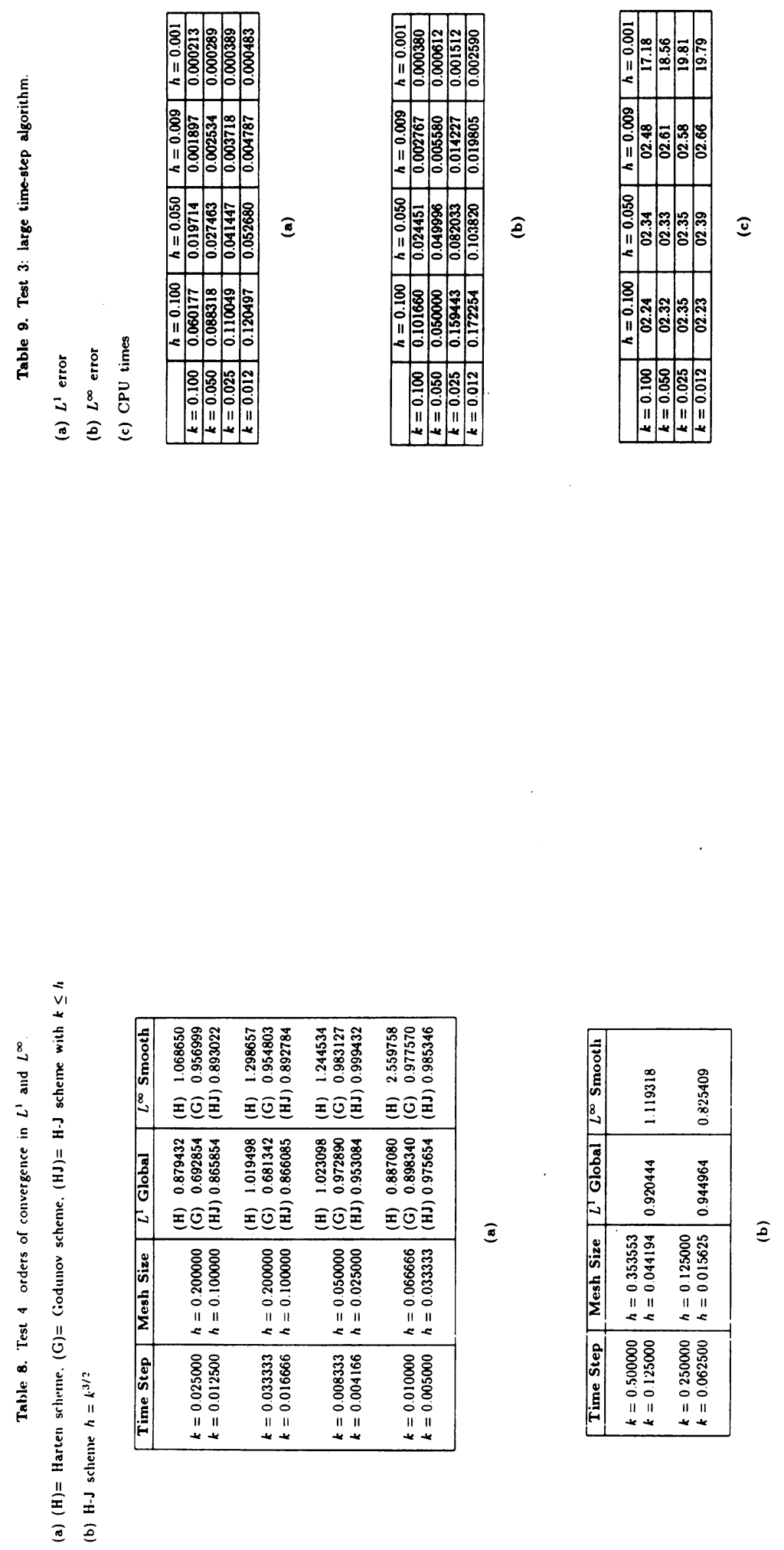

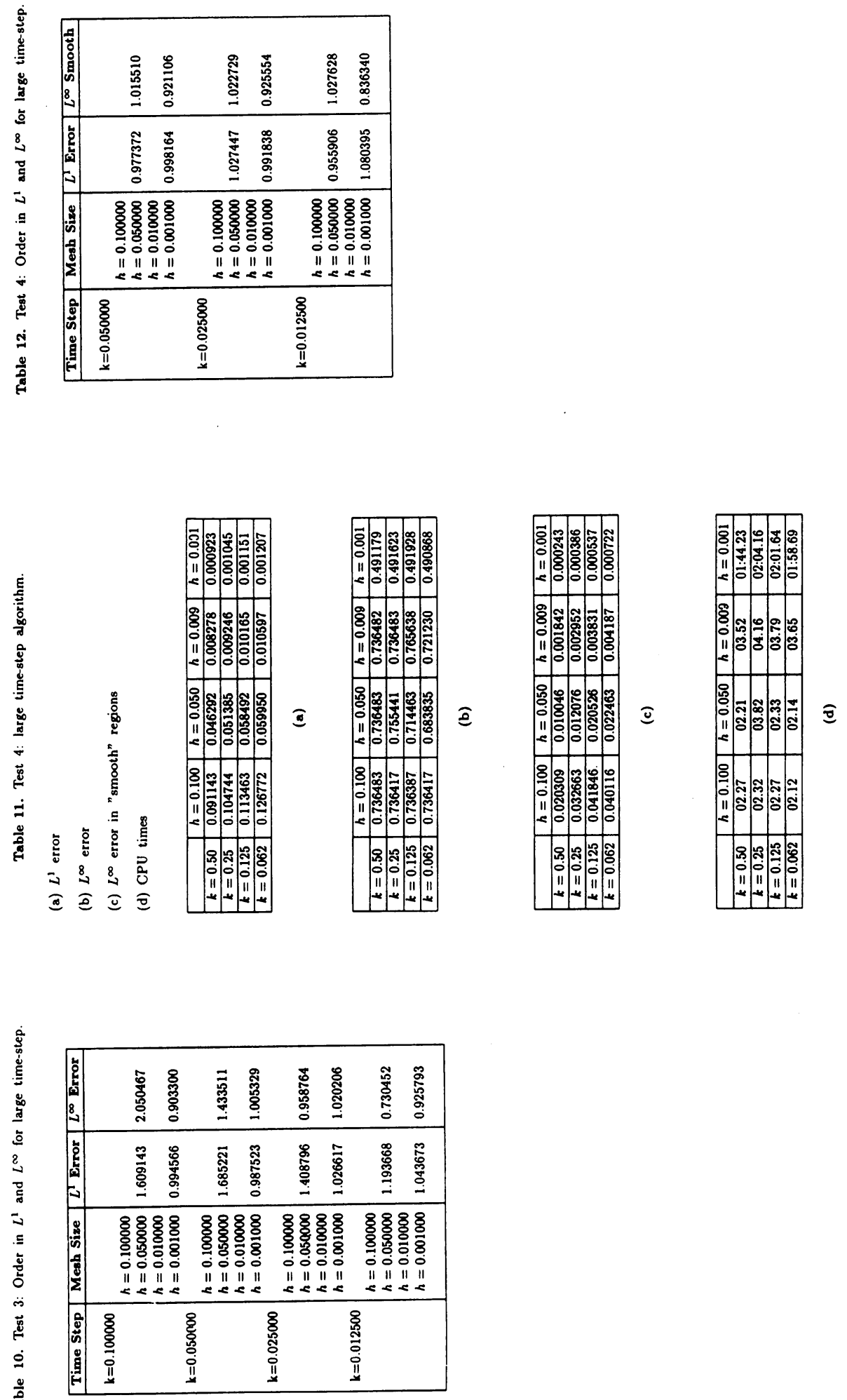